\title{
8 Was ist zu tun? Die Verankerung des Managements der sozialen Verantwortung im Krankenhaus
}

\subsection{Der Weg des Wandelns - ein Überblick}

Die verschiedenen Bausteine eines Konzepts des Managements der sozialen Verantwortung werden in den vorangegangenen Kapiteln beschrieben. Ein solches Konzept muss sich aber im Tun bewähren. Deshalb soll in einem abschließenden Kapitel - vor der Beschreibung eines praktischen Beispiels in Kapitel 9 -zusammenfasst werden, was die Geschäftsführung veranlassen muss, damit sozial verantwortliches Handeln zu einem festen Bestandteil des Arbeitsalltags aller Verantwortungsträger und aller Leistungsbereiche des Krankenhauses wird.

Die Neuausrichtung des Unternehmens bedeutet, die Goldene Regel als Ziel zu formulieren, um diese Grundhaltung in alle Entscheidungen einfließen zu lassen (Göbel 1992, S. 267). Die Neuausrichtung bedeutet ferner, die personellen Voraussetzungen dafür schaffen, dass die Verantwortungsträger Verantwortung wahrnehmen wollen und dieses auch können. Es kommt schließlich darauf an, die organisatorische Grundstruktur des Krankenhauses so zu gestalten, dass die Verantwortungsträger bei der Wahrnehmung ihrer sozialen Verantwortung unterstützt werden.

Wer dieses will, muss sich zunächst mit einer ungeschminkten und detaillierten Bestandsauf- nahme befassen, und zwar nicht nur, um die richtigen Veränderungsmaßnahmen veranlassen zu können. Die Standortbestimmung ist auch notwendig, um später feststellen zu können, was erreicht worden ist.

Diese Bestandsaufnahme fragt vor allem danach, - inwieweit das Management der sozialen Verantwortung oder einzelne Elemente davon schon praktiziert werden,

- welche personellen und institutionellen Voraussetzungen für das nachhaltige Realisieren des besseren Management-Konzepts bereits gegeben sind und

- welche Veränderungen gegebenenfalls noch veranlasst werden müssen.

Die Umstellung des Krankenhauses auf das bessere Management-Konzept ist auch dann ein radikaler Bruch mit über lange Zeit hinweg eingeübten und praktizierten und damit vertraut gewordenen Verhaltensweisen, wenn schon einige Elemente dieses Konzepts explizit und/oder implizit praktiziert werden. Es bedarf dennoch häufig grundlegender Veränderungen und diese flankierender Maßnahmen. Von diesen sind die Geschäftsführung und alle ihre Mitarbeiter sowie alle Organisationseinheiten des Krankenhauses betroffen. 
Dabei muss mit erheblichen Widerständen gerechnet werden. Die Gründe:

- Es gibt nur wenige praktische Erfahrungen mit derartigen Veränderungsprozessen, die genutzt werden könnten, einen solchen Prozess sozial verträglich und erfolgreich zu organisieren.

- Von den Verantwortungsträgern wird - was von der Geschäftsführung häufig unterschätzt wird - neben der Arbeitszeit für die Wahrnehmung ihrer Kernaufgaben sehr viel Zeit und Energie für die Umstellung auf das neue Management-Konzept gefordert.

- Zudem gibt es nicht wenige Mitarbeiter, die schlechte Erfahrungen mit der Reorganisation eines ganzen Unternehmens oder auch Teilen davon gemacht haben und der beabsichtigten Änderung deshalb eher mit Skepsis begegnen.

Damit die Umstellung auf das bessere Management-Konzept dennoch nicht scheitert, sind die sorgfältige Vorbereitung des Wandelns und das Beachten einiger Bedingungen, unter denen der Transformationsprozess realisiert wird, erforderlich. Von beidem soll in diesem Kapitel die Rede sein.

Bei der zusammenfassenden und ergänzenden Darstellung von Gestaltungsvorschlägen wird - wie auch schon in den Kapiteln 6 und 7 hervorgehoben, dass das Schaffen der organisatorischen und personellen Voraussetzungen zur Wahrnehmung von Verantwortung ein Prozess ist, der durch Grundpostulate des Managements der sozialen Verantwortung geleitet wird. Damit wird zweierlei erreicht:

1. Die organisationalen und personalen Barrieren, die sozial verantwortliches Handeln verhindern könnten, werden vermieden oder soweit diese existieren - beseitigt.

2. Mit der Erarbeitung von Vorschlägen für die Gestaltung des Managements der sozialen Verantwortung kann dieses trainiert und damit seine Akzeptanz vorbereitet werden.

Wenn hier und an anderen Stellen in diesem Buch immer wieder gefordert wird, das Unternehmensleitbild des Krankenhauses im Sinne der Grundidee des Managements der sozialen Verantwortung weiter zu entwickeln und danach konsequent zu praktizieren, heißt dieses nicht, dass von den Verantwortungsträgern in den Krankenhäusern angenommen wird, sie handelten bisher unverantwortlich. Im Gegenteil: Es wird unterstellt, dass es für viele Akteure im Krankenhaus ein Bedürfnis ist, die Goldene Regel zu praktizieren, dass es bisher aber nicht selten an den Voraussetzungen fehlt, die sie dabei unterstützen könnten, ihre Absichten in die Tat umzusetzen.

Der Weg dorthin wird zweckmäßigerweise in zwei Phasen/fünf Etappen zurückgelegt (s. Tab. 20; in Anlehnung an Berkel; Herzog, S. 79; Kotter, S. $55 f f$.$) .$

Die Etappen des Übergangs von einem konventionellen auf das bessere, weil sozial verantwortliches Handeln in den Vordergrund stellende Management-Konzept sind die Gegenstände dieses Kapitels. Dabei wird an mehreren Stellen auf die

Tab. 20 Die Phasen/Etappen des Wandelns

\section{Der Weg des Wandelns}

Phase I

Initiieren des Wandelns

\section{Phase II}

Strategien entwickeln für die nachhaltige Realisierung des Managements der sozialen Verantwortung
Management der sozialen Verantwortung als neues Management-Konzept beschließen (s. Kap. 8.2),

(damit sind sowohl der Grundsatzbeschluss gemeint, der den Veränderungsprozess auslöst, als auch der endgültige Beschluss mit vielen Festlegungen im Detail, der aber erst nach der Standortbestimmung möglich wird),

Standortbestimmung, die aus der Umwelt- und aus der Unternehmensanalyse besteht (s. Kap. 8.3 und 8.4),

die Übernahme von Verantwortung durch die Akteure des Krankenhauses konkretisieren (s. Kap. 8.6)

die Akteure des Krankenhauses für Verantwortungsbewusstsein sensibilisieren (s. Kap. 8.7) und

das Konzept des Managements der sozialen Verantwortung festigen (s. Kap. 8.8). 
Tab. 21 Der Weg des Wandelns

\section{Kapitel Inhalt des Kapitels}

8.2.1 Management der sozialen Verantwortung beschließen - Grundsatzbeschluss

Damit wird

Klarheit geschaffen über die Absichten der Geschäftsführung, die Vision des Wandelns vermittelt;

die Durchführung einer Bestandsaufnahme verfügt;

ein Gefühl für die Notwendigkeit, weil Nutzen stiftend, und für die Dringlichkeit des Wandelns erzeugt und

das für den radikalen Bruch mit bisher geübten Verhaltensweisen notwendige Arbeitsklima entwickelt.

8.2.2 Organisation des Veränderungsprozesses

Beauftragung des Projektmanagements;

Festlegen der Projekt-Organisation und als Teil davon

Sicherung der zur Umsetzung des Beschlusses erforderlichen Unterstützung durch Macht-Promotoren.

8.2.3 Management der sozialen Verantwortung endgültig beschließen und*

die Entwicklung bzw. Weiterentwicklung eines den Prinzipien der Goldenen Regel verpflichteten Unternehmensleitbildes beauftragen (s. auch Kap. 7.2);

Identifizierung und Veranlassen der Maßnahmen, die dem Schaffen der organisationalen Voraussetzungen für das Wahrnehmen von Verantwortung dienen (s. auch Kap. 6.4 und 6.5) und

Identifizierung und Veranlassen der Maßnahmen, die notwendig sind, um die Verantwortungsträger des Krankenhauses von der Nützlichkeit sozial verantwortlichen Handelns überzeugen und um diese in den Stand versetzen zu können, dieses auch zu praktizieren (s. auch Kap. 7.3 und 7.4).

* auf der Grundlage der Umwelt- und Unternehmensanalyse-Ergebnisse

8.3 Umweltanalyse auf Stakeholder-Basis

Welche Stakeholder sollen bei unternehmerischen Entscheidungen berücksichtigt werden?

Welche Stakeholder-Anliegen werden als legitim akzeptiert und bei Entscheidungen berücksichtigt?

$8.4 \quad$ Unternehmensanalyse

Wie ist die Einstellung der Verantwortungsträger gegenüber dem Praktizieren der Goldenen Regel?

Wie werden Stakeholder-Anliegen in der unternehmerischen Praxis berücksichtigt?

Wie sind die institutionellen und personellen Voraussetzungen für sozial verantwortliches Handeln in dem Krankenhaus beschaffen?

8.5 Wahrnehmung von Verantwortung konkretisieren durch

Festlegen der Regeln als Grundlage für die Formulierung und Einführung des Unternehmensleitbildes;

Festlegen der Werte und Normen, an denen sozial verantwortliches Handeln ausgerichtet werden soll und

Entwicklung bzw. Weiterentwicklung des Unternehmensleitbildes.

8.6 Für Verantwortungsbewusstsein sensibilisieren heißt

die Entscheidungsträger dafür gewinnen, die Anliegen der Stakeholder und die Folgen der Entscheidungen

für Dritte bei der Vorbereitung und dem Fällen ihrer Entscheidungen zu berücksichtigen.

8.7 Konzept des Managements der sozialen Verantwortung festigen durch

Evaluierung des Konzepts und der Handhabung des Managements der sozialen Verantwortung und

Sicherstellung des Wandelns durch kurzfristige Erfolge. 
detaillierte Beschreibung der Bausteine des Managements der sozialen Verantwortung in anderen Kapiteln verwiesen. Das gilt vor allem für die Gestaltung einiger Teilfunktionen betrieblicher Leitungstätigkeit und der Organisation der Leitungstätigkeit sowie für die Art der Realisierung der Entscheidungsprozesse (s. Kap. 6). Der Beantwortung der Frage, wie die Unterstützung der Verantwortungsträger gestaltet werden muss, damit diese Verantwortung wahrnehmen können, wurde in Kapitel 7 die notwendige Aufmerksamkeit gewidmet, sodass auch darauf zurückgegriffen werden kann und sich auch hierzu detaillierte Ausführungen in diesem Kapitel erübrigen.

Andere Etappen des Weges zu dem besseren Management-Konzept bedürfen einer ausführlicheren Darstellung. Das gilt vor allem für die Charakterisierung der Stakeholder und die Analyse der Stakeholder-Anliegen sowie für die Feststellung, welche Bedeutung die Stakeholder für das Krankenhaus haben und mit welchem Gewicht die Stakeholder-Bedürfnisse bei Entscheidungen berücksichtigt werden sollen (s. Kap. 8.3 - Umweltanalyse auf Stakeholder-Basis). Das gilt auch für die Beantwortung der Frage, für wie bedeutsam die Akteure des Krankenhauses die Realisierung des Managements der sozialen Verantwortung halten und welchen Nutzen sie für das Krankenhaus und für sich selbst als Folge der Realisierung des neuen Management-Konzepts erwarten (s. Kap. 8.4 - Unternehmensanalyse). Die Beantwortung dieser Fragen steht im Zentrum der vorzunehmenden Standortbestimmung.

Die Notwendigkeit zu einer etwas ausführlicheren Darstellung ergibt sich auch für die Etappen der Phase II. Es werden Strategien in Anlehnung an Methoden der Einstellungsentwicklung und Organisationsentwicklung vorgestellt, die dazu beitragen sollen, sozial verantwortliches Handeln im Sinne der Goldenen Regel zum bewussten Teil des Arbeitsalltags im Krankenhaus zu machen (Berkel; Herzog, S. 79). Es werden Vorschläge für das Konkretisieren der Wahrnehmung von Verantwortung und für das Sensibilisieren der Verantwortungsträger für die Nützlichkeit des Konzepts sowie für die Festigung des Konzepts präsentiert. Einige Aussagen aus den Kapiteln 6 und 7 werden in diesem Zusammenhang noch einmal aufgenommen; sie werden ergänzt und detailliert. Einige kleinere Wiederholungen sind deshalb nicht ganz ausgeschlossen.

Der Weg des Wandelns ist mit der Implementierung des neuen Management-Konzepts nicht beendet. Mit Hilfe der regelmäßigen Evaluierung des Konzepts - sie ist eines der Instrumente, die der Festigung des Konzepts dienen - gilt es herauszufinden,

- wie die Verantwortungsträger die Normen des Unternehmensleitbildes praktizieren und ob Letzteres gegebenenfalls veränderten Umweltund Unternehmensbedingungen angepasst werden muss, damit das neue ManagementKonzept dauerhaft mit dem gewünschten Erfolg umgesetzt werden kann und

- ob die personellen und institutionellen Voraussetzungen so beschaffen sind, dass das Wahrnehmen von Verantwortung unterstützt wird.

Des bessern Überblicks wegen ist der Weg des Wandelns in der Tabelle 21 in Stichworten zusammengestellt. Dabei handelt es sich um ein erstes, nicht vollständiges Zwischenergebnis. Eine $\mathrm{Zu}$ sammenfassung aller Transformationsmaßnahmen erfolgt am Ende dieses Kapitels.

\subsection{Management der sozialen Verantwortung beschließen}

\subsubsection{Grundsatzbeschluss}

Ausschlaggebend für die erfolgreiche Umstellung auf das Management der sozialen Verantwortung ist, dass die Initiative für die Umstellung von der Geschäftsführung ausgeht und dass das bessere Management-Konzept dauerhaft von dieser getragen wird. Die Geschäftsführung macht mit einem Grundsatzbeschluss deutlich, dass es eine ihrer zentralen Aufgaben ist, die Entwicklung und die Implementierung des neuen Management-Konzepts sowie das Schaffen der Voraussetzungen für das nach haltige Wahrnehmen von Verantwortung durch alle Verantwortungsträger des Krankenhauses zu initiieren und zu tragen. Sie verspricht mit dem Crundsatzbeschluss, dass sie sich vordringlich und für alle anderen Verantwortungsträger erkennbar dieser Aufgabe widmen wird. Die Geschäftsführung gibt als Vorbild ihren Mitarbeitern Gelegenheit, verantwortungsbewusstes Handeln zu lernen und zu lernen, mit der Freiheit, die ihnen eingeräumt werden soll, verantwortlich umzugehen.

Mit dem förmlichen Grundsatzbeschlusses, dass das neue Management-Konzept eingeführt werden soll, schafft die Geschäftsführung im Sin- 
ne einer Top-down-Planung Klarheit darüber, was sie konkret will. Sie formuliert die Ziele, die mittels des besseren Management-Konzepts realisiert werden sollen, und legt dem Grunde nach fest, was sie von ihren Mitarbeitern künftig im Sinne des Managements der sozialen Verantwortung erwartet.

Mit dem Grundsatzbeschluss verfügt die Geschäftsführung eine sorgfältige Standortbestimmung. Sie macht die Notwendigkeit und die Dringlichkeit des Wandelns deutlich (Kotter, S. 55 ff.). Sie verweist dabei auf die Ergebnisse einer aktuellen, die Stakeholder-Analyse allerdings nicht umfassenden, Umweltanalyse und auf die darin erkennbaren Chancen für eine erfolgreiche Weiterentwicklung des Krankenhauses. Sie belegt mit den Ergebnissen einer aktuellen Unternehmensanalyse, dass das Krankenhaus nicht darauf vorbereitet ist, diese Chancen wahrzunehmen.

Wenn damit der Nachweis für die Dringlichkeit der Veränderung nicht erbracht werden kann - weil die Selbstzufriedenheit der Verantwortungsträger mit ihrer gegenwärtigen Situation zu hoch ist -, werden - eventuell in einem Begleitdokument zu dem Crundsatzbeschluss Maßnahmen festgelegt, deren Realisierung geeignet ist, das Bewusstsein für die Dringlichkeit des Wandelns zu steigern (s. Beispiel 1 - weitere Beispiele dazu siehe bei Kotter, S. 67).

\section{Beispiel 1}

Die Geschäftsführung eines Krankenhauses verfügt das Eliminieren sichtbarer Beispiele für Verschwendung:

- die große Flotte sehr aufwändiger Dienstfahrzeuge wird abgeschafft,

- das Großgerät, das vorwiegend der Befriedigung des Egos des Leiters einer klinischen Abteilung dient und bei weitem nicht ausgelastet sein wird, wird nicht beschafft, und

- die Leiter verschiedener klinischer Abteilungen werden verpflichtet, vorhandene Ressourcen gemeinsam zu nutzen.

Die Geschäftsführung beauftragt das Projektmanagement, das den Prozess der Einführung des Managements der sozialen Verantwortung im Detail plant und steuert.

Mit diesem Grundsatzbeschluss sollte ein Arbeitsklima geschaffen werden können, in dem Freude an der Wahrnehmung sozialer Verantwor- tung begründet wird. Das wird erreicht, indem glaubhaft gemacht wird, dass künftig von allen Verantwortungsträgern des Krankenhauses verlangt und dass es ihnen aber auch ermöglicht wird, unternehmerische Entscheidungen an den Grundpostulaten des Managements der sozialen Verantwortung auszurichten.

\subsubsection{Organisation des Veränderungsprozesses}

Wie jeder Veränderungsprozess bedarf auch die Umstellung des Krankenhauses auf das Konzept des Managements der sozialen Verantwortung einer straffen Organisation. Von zentraler Bedeutung in diesem Zusammenhang ist die Installation eines professionellen Projektmanagements und die Verpflichtung eines Projektverantwortlichens, der der Geschäftsführung direkt berichtet.

Hinsichtlich der Einzelheiten zu der Organisationsentwicklung wird auf die zahlreiche Spezialliteratur verwiesen. Nur so viel an dieser Stelle:

Um ein so ambitioniertes Projekt wie die Einführung eines neuen Management-Konzepts schultern zu können, bedarf die Geschäftsführung der Unterstützung durch geeignete MachtPromotoren. Diese haben die Aufgabe, zusammen mit der Geschäftsführung für das Management der sozialen Verantwortung zu werben und dem neuen Management-Konzept damit zur nachhaltigen Wirksamkeit zu verhelfen. Zu diesen Macht-Promotoren zählen im Regelfall die Mitglieder der Leitungsebene, die der Ebene der Geschäftsführung unmittelbar nachgeordnet ist. $\mathrm{Zu}$ den Macht-Promotoren zählen auch die Personalvertretungen. Wichtig ist, dass die genannten Führungskräfte und die Mitglieder der Personalvertretung möglichst ohne Ausnahme bereit sind, das neue Unternehmensleitbild zu akzeptieren und zu praktizieren. Beide Gruppen werden dadurch gewonnen, indem mit ihnen gemeinsam die Eckdaten für das neue Management-Konzept, die Gegenstand des Grundsatzbeschlusses sind, entwickelt werden.

Zusätzlich zu diesen mehr repräsentativ wirkenden Macht-Promotoren benötigt die Geschäftsführung - zumindest in größeren Unternehmen - ein Führungsteam, das ihr hilft, den Wandel inhaltlich vorzubereiten und anschließend zu realisieren (Kotter, S. 75ff.). Die richtige Zusammensetzung dieses Teams ist für die erfolg- 
reiche Realisierung des Veränderungsprozesses von zentraler Bedeutung (Kotter, S. 83):

- Es bedarf mindestens eines Mitgliedes, das bei den potenziellen Macht-Promotoren die notwendige Autorität genießt, um diese gemeinsam mit der Geschäftsführung davon überzeugen zu können, möglichst vollständig und gemeinsam im Sinne der Geschäftsführung zu agieren.

- Die Mitglieder des Führungsteams verfügen über die sozialen Kompetenzen, um die Verantwortungsträger motivieren zu können, sich an dem Veränderungsprozess aktiv zu beteiligen.

- Das Führungsteam verfügt über die Sachkenntnis, um die notwendigen Entscheidungen sachgerecht vorbereiten oder selbst fällen zu können.

- Die Mitglieder des Führungsteams genießen innerhalb des Unternehmens großes Vertrauen und können auf dieser Crundlage alle Verantwortungsträger von der Vorteilhaftigkeit des Managements der sozialen Verantwortung überzeugen. Sie dienen als Ansprechpartner für alle Verantwortungsträger.

Die Umstellung von dem bisherigen auf das bessere Management-Konzept wird in jedem Krankenhaus, und zwar unabhängig von der konkreten Ausgangssituation, mehrere Jahre in Anspruch nehmen. Auf der anderen Seite ist es - um die Mitarbeiter von der Vorteilhaftigkeit des neuen Management-Konzepts überzeugen zu können wichtig, dass möglichst rasch die ersten Erfolge vorzeigbar sind. Es empfiehlt sich deshalb, den Veränderungsprozess in Teilprozesse mit jeweils einem eindeutig definierten und für sich alleine nutzbaren Ergebnis zu zerlegen. Der eine oder andere Teilprozess sollte möglichst rasch umgesetzt werden (können) (s. Beispiel).

\section{Beispiel}

Die Geschäftsführung erlässt - als Teil umfassender Personalmanagement-Grundsätze - eine neue Richtlinie für die Durchführung der Personalbedarfsbestimmung. Sie legt darin fest, dass die Mitarbeiter der Leistungsbereiche in dem als Gegenstromverfahren organisierten Prozess zu dem für sie relevanten Entwurf der Personalbedarfsbestimmung mindestens gehört werden, bevor die sie betreffenden Entscheidungen gefällt werden, und dass - wenn deren Anregungen nicht berücksichtigt werden (können) - dieses von dem Entscheidungsträger plausibel begründet wird. Das Ergebnis der Personalbedarfsbestimmung für jeweils einen Leistungsbereich wird im Rahmen einer Zielvereinbarung zwischen der Geschäftsführung und dem für den Leistungsbereich Verantwortlichen verbindlich gemacht.

Die Richtlinie lässt erkennen, welchen Anforderungen die Verantwortungsträger künftig genügen müssen, um mit diesem Planungsinstrument umgehen zu können. Sie nennt Maßnahmen der Personalentwicklung, mit deren Hilfe sich die Verantwortungsträger auf das Umsetzen der Richtlinie vorbereiten können.

Wenn der Prozess der Personalbedarfsermittlung wie skizziert organisiert wird, ist er durch mehrere Grundpostulate geleitet:

- Unter anderem werden die Interessen der Betroffenen - das sind vor allem die Patienten, die Führungskräfte, die Mitarbeiter sowie auch der Eigentümer und die Krankenkassen - berücksichtigt.

- Die Personalausstattung ist das Ergebnis einer argumentativen, dialogischen Verständigung zwischen der Geschäftsführung und den Betroffenen.

- Der Prozess genügt der Begründungspflicht.

Das Erarbeiten einer solchen Richtlinie nimmt nicht viel Zeit in Anspruch; die Geschäftsführung kann mit deren Umsetzen die Verantwortungsträger rasch von der Vorteilhaftigkeit sozial verantwortlichen Handelns überzeugen.

\subsubsection{Das Wandeln endgültig beschließen - mit der Umstellung beginnen}

Mit dem endgültigen Umstellungsbeschluss wird festgelegt, welche Maßnahmen realisiert werden müssen, damit die Verantwortungsträger Verantwortung ohne Einschränkung wahrnehmen können. Die Analyse der Ausgangssituation (= Standortbestimmung) - sie ist Gegenstand der Kapitel 8.3 und 8.4 - liefert die dafür notwendigen Informationen.

Als Ergebnis einer Umweltanalyse auf Stakeholder-Basis (s. Abb. 4 und Kap. 8.3) zeigt sie, welche Stakeholder wegen ihrer Beiträge für das Krankenhaus und wegen der Stärke ihres Einflusses wie bedeutsam für das Krankenhaus sind und welche Anliegen in Abhängigkeit davon es mittels unternehmerischer Entscheidungen zu befriedigen gilt. Es wird deutlich, mit welchem Gewicht die Stakeholder-Bedürfnisse bei Entscheidungen berücksichtigt werden sollten.

Auf der Crundlage der Ergebnisse der Unternehmensanalyse (s. Abb. 5 und Kap. 8.4) lässt sich feststellen, wie wichtig den Verantwortungsträgern das Praktizieren der Coldenen Regel aktuell 
ist und ob überhaupt und inwieweit den Anforderungen, die in Kapitel 6 (der institutionelle Rahmen für die Wahrnehmung von Verantwortung) und Kapitel 7 (die Unterstützung der Verantwortungsträger bei der Wahrnehmung von Verantwortung) erörtert worden sind (= Soll-Zustand), bereits entsprochen wird (= Ist-Zustand). Möglicherweise bestehen noch Differenzen zwischen dem als Basis für die Realisierung des Managements der sozialen Verantwortung angestrebten Soll-Zustand und dem Ist-Zustand, die Gestaltung einiger zentraler Teilfunktionen betrieblicher Leitungstätigkeit, die Organisation der Leitungstätigkeit, die Gestaltung der Entscheidungsprozesse sowie die Unterstützung der Verantwortungsträger bei der Wahrnehmu $\neq$ ng von Verantwortung betreffend. Der endgültige Beschluss greift diese Defizite auf und benennt die Maßnahmen, die erforderlichenfalls umgesetzt werden müssen, um eventuell existierende und die Wahrnehmung von Verantwortung behindernde Barrieren beseitigen zu können und den Akteuren im Krankenhaus sozial verantwortliches Handeln nachhaltig zu ermöglichen.

Die Standortbestimmung liefert die Gründe für die Dringlichkeit der Umstellung auf das neue Management-Konzept - oder anders gesagt: sie dient dem Abbau der Zufriedenheit mit einem eventuell unzureichenden Ist-Zustand und bereitet damit den Boden für die notwendigen Änderungen. Vor allem das unvoreingenommene Gespräch mit den externen Stakeholdern lässt die Notwendigkeit und die Art personeller und institutioneller Veränderungen sowie deren Dringlichkeit deutlich werden (Kotter, S. 74).

Sobald die Ergebnisse der Standortbestimmung vorliegen, werden

- die Entwicklung bzw. Weiterentwicklung eines den Prinzipien der Goldenen Regel verpflichteten Unternehmensleitbildes, dessen Formulierung und Diffusion.

- das Identifizieren und das Veranlassen der Maßnahmen, die dem Schaffen der organisationalen Voraussetzungen für das Wahrnehmen von Verantwortung dienen und

- das Identifizieren und das Veranlassen der Maßnahmen, die notwendig sind, um die Verantwortungsträger des Krankenhauses von der Nützlichkeit sozial verantwortlichen Handelns überzeugen und in den Stand versetzen zu können, dieses auch zu praktizieren beauftragt.

\begin{abstract}
Aufbauend auf den Ergebnissen der Umwelt- und der Unternehmensanalyse wird die Umstellung nunmehr endgültig in Gang gesetzt. Einen Weg zurück gibt es jetzt nicht mehr.
\end{abstract}

\subsection{Umweltanalyse auf Stakeholder-Basis}

\subsection{1 Überblick und Einführung}

Stakeholder gehen Koalitionen mit Krankenhäusern ein, weil sie sich davon die Befriedigung bestimmter Bedürfnisse versprechen. Koalitionen werden auch deshalb eingegangen, weil Interessenten eigene Beiträge zur Realisierung der Unternehmensziele leisten wollen (Staehle, S. 431). Welche der möglicherweise sehr vielen Stakeholder, die eine Befriedigung ihrer Bedürfnisse erwarten, mit welchen Bedürfnissen bei Entscheidungen der Verantwortungsträger berücksichtigt werden sollen, wird als Ergebnis der Umweltanalyse festgestellt.

Diese wird in zwei Schritten durchgeführt:

1. Auswahl der weiter zu berücksichtigenden Stakeholder (s. Kap. 8.3.2)

Das Ergebnis dieses Analyse-Schrittes ist die Bedeutung der Stakeholder für das Krankenhaus. Anhand dieser erfolgt die Auswahl der Stakeholder aus einer möglicherweise größeren Zahl relevanter Stakeholder, die bei dem nächsten Analyse-Schritt berücksichtigt werden soll.

Die Auswahl erfolgt anhand zweier Kriterien:

- Zunächst wird geprüft, wie wichtig dem Krankenhaus die von den Stakeholdern zu erwartenden Beiträge sind und welche weiteren Bedingungen der Zusammenarbeit zwischen dem Krankenhaus und diesen Stakeholdern von dem Verantwortungsträger gegebenenfalls zu beachten sind. Die Ergebnisse werden je Stakeholder in einer Kennzahl „Wichtigkeit der StakeholderBeiträge" zusammengefasst.

- Des Weiteren wird festgestellt, welcher Art und wie stark der Einfluss ist, den die Stakeholder zwecks Durchsetzen ihrer Anliegen geltend machen können. Die Ergebnisse werden je Stakeholder in einer Kennzahl „Stärke des Stakeholder-Einflusses“ festgehalten. 
- Die beiden Kennzahlen werden zu einer Kennzahl „Bedeutung des Stakeholders“ kombiniert.

2. Auswahl der zu berücksichtigenden Stakeholder-Bedürfnisse (Kap. 8.3.3).

Die Umweltanalyse wird fortgesetzt mit der Identifizierung und Analyse der Anliegen der im ersten Analyse-Schritt ausgewählten Stakeholder. Diese werden bewertet, um entscheiden zu können, welche Bedürfnisse mit welchem Gewicht bei den Entscheidungen der Verantwortungsträger berücksichtigt werden sollen.

Es kommt darauf an, dass die Geschäftsführung und die Verantwortungsträger bei der Sammlung der für die Durchführung dieses Auswahlprozesses erforderlichen Informationen unvoreingenommen und offen agieren. Nicht selten bereiten aber der Geschäftsführung sowie deren Mitarbeitern die Identifizierung der Stakeholder, deren Charakterisierung und schließlich die Analyse und Bewertung der Stakeholder-Anliegen und -Beiträge in der Praxis große Schwierigkeiten. Häufig sehen sich die Verantwortungsträger im Krankenhaus auf Grund ihrer Rolle ausschließlich für die Befriedigung der Patienten-, der Mitarbeiter- und der Eigentümerbedürfnisse verantwortlich und nehmen deshalb andere Stakeholder und vor allem deren Anliegen und deren Möglichkeiten, unternehmerische Entscheidungen zu beeinflussen, nicht wahr. Die Unternehmen konzentrieren sich nicht selten nur auf die Befriedigung ihrer eigenen Bedürfnisse (PWC, S. 3).

Dies liegt vor allem daran, dass es für die Geschäftsführung und insbesondere für die Vertreter der Leitungsebene unterhalb der Geschäftsführungs-Ebene (wie z.B. für Chefärzte und für Leitende Pflegekräfte) ungewohnt ist, das Unternehmen von außen zu sehen und sich in die Lage derer zu versetzen, die von ihren Entscheidungen betroffen sind. Die leitenden Mitarbeiter vor allem die des ärztlichen, des Pflege-Dienstes und die der para-medizinischen Dienste sehen im Regelfall - weil sie nur dieses während ihrer Ausbildung und in der daran anschließenden Praxis gelernt haben - ausschließlich die Anliegen der Patienten und vielleicht noch die ihrer Mitarbeiter.

Von der Geschäftsführung werden häufig nur noch jene Tatbestände, die krankenhausintern mit den Behandlungs-, Support- und Betriebsführungsprozessen unmittelbar im Zusammenhang stehen, als betrachtungswürdig angese- hen. Andere Tatbestände werden - nicht selten als Ergebnis einer eher eingeschränkten Sichtweise und die Folgen für das Krankenhaus nicht bedenkend - als für den Betrieb des Krankenhauses nicht relevant identifiziert und deshalb ausgegrenzt (s. Beispiel 1).

\section{Beispiel 1}

Ein Krankenhaus ist darauf angewiesen, dass in seiner unmittelbaren Nachbarschaft Handwerks- und Dienstleistungsunternehmen angesiedelt sind, die im Bedarfsfall jederzeit und schnell zur Verfügung stehen. Dabei handelt es sich meist um kleinere und mittelständische Unternehmen, die für ihre Leistungen auf Grund ihrer Kostenstruktur nicht selten höhere Preise verlangen müssen als große (Konzern-)Unternehmen, die meist aber sehr viel schneller als die großen Dienstleister und Lieferanten einsatzfähig sind.

Nicht wenige Krankenhaus-Geschäftsführungen zählen die Existenzsicherung der zuerst genannten Unternehmen nicht zu ihren Aufgaben und sind deshalb auch nicht bereit, einen Preisaufschlag zu akzeptieren. Wenn deshalb ein solches Unternehmen vom Markt verschwinden muss, wirkt sich dieses möglicherweise nachteilig auf die Qualität und die Sicherheit der Patientenbehandlung aus.

Die Umweltanalyse auf Stakeholder-Basis ist auch deshalb so wichtig, weil die Gestaltung der personellen und institutionellen Voraussetzungen für die angemessene Berücksichtigung der Stakeholder-Anliegen bei unternehmerischen Entscheidungen entscheidend von der Art der zu berücksichtigenden Stakeholder, deren Anliegen und deren Möglichkeiten des Einflusses auf das Verantwortungsträger-Handeln abhängt (s. Beispiel 2).

\section{Beispiel 2}

Wenn die Personalbedarfsermittlung ausschließlich auf die Befriedigung der Eigentümer-Bedürfnisse ausgerichtet werden müsste, dann wäre die Organisation des Prozesses der Personalbedarfsermittlung vernünftigerweise eine andere als die weiter oben beispielhaft skizziert. Das eher zeitaufwändige Gegenstromverfahren könnte durch eine sehr viel weniger aufwändige Top-down-Planung ersetzt werden.

Die Analysten sollten sich auf jene Stakeholder und Stakeholder-Anliegen konzentrieren, von denen angenommen werden kann, dass deren 
Verbindung zu dem Krankenhaus nicht nur spontaner, sondern dauerhafter Natur ist. Die notwendige und meist sehr aufwändige Analyse der Stakeholder und ihrer Anliegen sowie deren Gewichtung und die Bewertung der Stakeholder-Bedürfnisse als Basis für das nachhaltige Praktizieren der Goldenen Regel lässt sich nur dann rechtfertigen, wenn die Analyse-Ergebnisse zumindest mittelfristig Bestand haben können.

\subsubsection{Auswahl der zu berücksichtigenden Stakeholder}

\section{Identifizierung der Stakeholder}

Es sollte zunächst ein möglichst umfassender Katalog von zu berücksichtigenden Stakeholdern erstellt werden. Darin sollten auch jene Stakeholder einbezogen werden, die auf den ersten Blick für das Krankenhaus und/oder einzelne seiner
Leistungsbereiche vielleicht nicht relevant sind. An der Erarbeitung der Stakeholder-Liste sollten möglichst alle Verantwortungsträger beteiligt werden, um der Gefahr entgehen zu können, dass der eine oder andere Stakeholder, der zu den Betroffenen unternehmerischer Entscheidungen zählt, übersehen wird.

Das Identifizieren der Adressaten von Verantwortung ist ein äußerst komplexer Vorgang. Die Lösung dieses Problems wird etwas vereinfacht, wenn man die Umwelt, in der die Stakeholder verortet sind, zunächst in Sektoren aufteilt (s. Tab. 22) (Göbel 1992, S. 147ff.). Die Identifizierung der Stakeholder in diesen Sektoren wird einzelnen Experten - so z.B. die der Stakeholder innerhalb des Sektors „Kapital-/Geldgeber“ dem Leiter der Finanzabteilung - zugewiesen. Die Gefahr, dass relevante Stakeholder übersehen werden, wird damit geringer.

Die Umwelt des Krankenhauses könnte - ausgehend von der Zusammenstellung der Stakehol-

Tab. 22 Umweltsektoren und Stakeholder (Beispiele)

\begin{tabular}{|c|c|c|c|}
\hline Staat und Öffentlichkeit & Patienten & Arbeitnehmer & Kapital-/Geldgeber \\
\hline $\begin{array}{l}\text { Ministerien } \\
\text { andere Behörden } \\
\text { Verbände } \\
\text { Kammern } \\
\text { Parteien } \\
\text { Medien } \\
\text { Anrainer } \\
\text { Kirchen } \\
\text {... }\end{array}$ & $\begin{array}{l}\text { Patienten der Gesetzlichen } \\
\text { Krankenversicherung } \\
\text { Patienten der Privaten } \\
\text { Krankenversicherung } \\
\text { selbstzahlende Patienten } \\
\text { potenzielle Patienten } \\
\text {.... }\end{array}$ & $\begin{array}{l}\text { vorhandene Mitarbeiter, } \\
\text { gegebenenfalls gegliedert } \\
\text { nach diversen Kriterien } \\
\text { Führungskräfte } \\
\text { Chefärzte } \\
\text { potenzielle Mitarbeiter } \\
\text { Auszubildende } \\
\text { Praktikanten } \\
\text { Personalvertretung } \\
\text {... }\end{array}$ & $\begin{array}{l}\text { Eigentümer } \\
\text { Ministerium (Fördergelder) } \\
\text { Banken } \\
\text { Krankenkassen } \\
\text { Sponsoren } \\
\text {... }\end{array}$ \\
\hline Kapital-/Geldgeber & andere Kunden & Wettbewerber & Lieferanten \\
\hline $\begin{array}{l}\text { Eigentümer } \\
\text { Ministerium (Fördergelder) } \\
\text { Banken } \\
\text { Krankenkassen } \\
\text { Sponsoren } \\
\text {... }\end{array}$ & $\begin{array}{l}\text { Angehörige } \\
\text { Besucher } \\
\text { einweisende Ärzte } \\
\text { Selbsthilfegruppen } \\
\text { Anbieter, die medizinische } \\
\text { Leistungen der Diagnostik } \\
\text { (wie z.B. Befunde der } \\
\text { Laboratoriumsmedizin) und } \\
\text { anderes mehr anbieten } \\
\text {... }\end{array}$ & $\begin{array}{l}\text { andere Krankenhäuser } \\
\text { sonstige Anbieter } \\
\text { medizinischer Leistungen } \\
\text {... }\end{array}$ & $\begin{array}{l}\text { Warenlieferanten } \\
\text { unter anderem für } \\
\text { Arzneimittel und sonstige } \\
\text { Medizinalprodukte, } \\
\text { medizin-technische Geräte } \\
\text { Dienstleister } \\
\text { Beratungsunternehmen } \\
\text {... }\end{array}$ \\
\hline
\end{tabular}


der in Tabelle 4 - beispielsweise und als Ausschnitt wie folgt gegliedert werden (s. Tab. 22) (Straub, S. 25).

\section{Die Bedeutung der Stakeholder für das Krankenhaus}

Um am Ende des Analyseprozesses die (wenigen) Stakeholder auswählen zu können, die als relevant für den folgenden Analyse-Schritt angesehen werden und deren Berücksichtigung im Rahmen des Konzepts der sozialen Verantwortung bewältigt werden kann, ist die Feststellung der Bedeutung der Stakeholder für das Krankenhaus erforderlich. Diese wird von zwei Faktoren beeinflusst:

- von der Wichtigkeit der Beiträge, die Stakeholder als Basis für die Realisierung der Krankenhausziele leisten, und

- von der Art und der Stärke des Einflusses, mit dem die Stakeholder die Befriedigung ihrer Bedürfnisse geltend machen.

Bei der Ermittlung der Stakeholder-Bedeutung muss darauf geachtet werden, dass bei nicht wenigen Stakeholdern die beiden Einflussfaktoren gemeinsam-z.B. als Leistung des Krankenhauses und als Gegenleistung des Stakeholders - wirken (s. Abb. 32).

\section{a) Die Wichtigkeit der Stakeholder-Beiträge für das Krankenhaus}

Es gibt eine Vielzahl von Individuen und Institutionen, die aus unterschiedlichen Gründen ein Interesse an der Sicherung der langfristigen Existenz, der Weiterentwicklung sowie an der Steigerung der Qualität der Krankenhausleistungen und der Effizienz der Leistungserstellung haben (Cyert; March, S. 27ff.; Staehle, S. 427 und die dort angegebene Literatur). Sie versuchen deshalb, mit Anregungen und Initiativen die Entscheidungen der Geschäftsführung in diesem Sinne zu beeinflussen. Sie erwarten - gewissermaßen als Gegenleistung -, dass ihren Bedürfnissen durch unternehmerisches Handeln Rechnung getragen wird.

Auch für die Identifizierung der StakeholderBeiträge gilt, dass als Grundlage für die weiteren Analyse-Schritte eine möglichst vollständige Liste erstellt wird. In diese Liste sollten auch solche Beiträge aufgenommen werden, von denen zunächst nicht sicher ist, ob sie für die Ermittlung der Stakeholder-Bedeutung wirklich relevant sind. Fragen allgemeiner Art, wie sie im ersten Teil der folgenden Checkliste beispielhaft formuliert sind, können für die Erarbeitung einer möglichst vollständigen Liste von Stakeholder-Beiträgen eine Hilfe sein.

\section{Checkliste für die Analyse der Stakeholder-Beiträge (Ausschnitt)}

\section{Fragen nach zu erwartenden Stakeholder-Beiträgen allgemeiner Art}

- Welche Beiträge leisten die Interessenten, die geeignet sind, die Realisierung der von der Geschäftsführung verfolgten Ziele zu unterstützen, gegebenenfalls auch zu unterlaufen?

- Mit welchen Anregungen und Initiativen versuchen Stakeholder, Einfluss zu nehmen auf die Gestaltung und die Realisierung der Unternehmensziele?

-

\section{Stakeholder/Stakeholder-Beiträge}

\section{Patient}

- Patienten der Gesetzlichen Krankenversicherung entrichten Krankenkassenbeiträge, die von den Krankenkassen genutzt werden, um für die Patienten die der Art der Behandlungen entsprechenden Fallpauschalen zu entrichten;

- selbst zahlende Patienten entrichtet die Fallpauschale für die in Anspruch genommene Leistung;

- Patient empfiehlt das Krankenhaus weiter;

- ...

\section{Niedergelassene Ärzte}

- weisen die von innen ambulant behandelten Patienten bei Bedarf in das Krankenhaus zur stationären Weiterbehandlung ein;

- erarbeiten zusammen mit den Ärzten des Krankenhauses Behandlungsleitlinien für ausgewählte Krankheitsarten, die die ambulante sowie die stationäre Behandlung umfassen;

-

\section{Mitarbeiter und Führungskräfte}

- stellen ihre Arbeitskraft, ihre Fähigkeiten und Kenntnisse dem Krankenhaus zur Verfügung;

- sind zu besonderen Anstrengungen bereit, um die Qualität und die Effizienz der Behandlungs-, Support- und Betriebsführungsprozesse kontinuierlich weiter verbessern zu können;

- entwickeln Behandlungsmethoden weiter und tragen damit zu der Steigerung des Krankenhaus-Images bei;

- halten Kontakt unter anderem zu Unternehmen der Pharma- und der Medizintechnikindustrie, um mit die- 
sen gemeinsam Entwicklungsarbeiten durchführen zu können;

- repräsentieren das Krankenhaus bei Berufsverbänden, Kammern, Kongressen usw. und tragen auch auf diese Weise zur Verbesserung des Krankenhaus-Images und des Images ihrer Berufsgruppe bei;

- halten Kontakt zu Ausbildungsstätten (unter anderem zu Universitäten, Hochschulen und Krankenpflegeschulen) und helfen damit bei der Suche nach qualifizierten und motivierten Mitarbeitern;

- beteiligen sich an der Öffentlichkeitsarbeit des Krankenhauses und tragen damit zur Steigerung des Bekanntheitsgrades des Krankenhauses bei;

- erbringen ihre Leistungen auf der Grundlage moralischer Prinzipien - wie z.B. Menschenwürde, Freiheit, Solidarität, Vermeiden von Verschwendung;

- sind gegenüber dem Patienten und dem Arbeitgeber loyal;

-..

\section{Krankenkassen}

- stellen die finanziellen Ressourcen zur Verfügung, die zur Deckung der durch die Behandlung der Patienten verursachten Kosten benötigt werden;

- .

\section{Eigentümer}

- überlassen das für die finanzielle Sicherung des Krankenhauses notwendige Kapital;

- unterstützen das Schaffen leistungsfähiger Managementstrukturen;

-.

Außer der Wichtigkeit der Beiträge, die Stakeholder für die Realisierung der Krankenhausziele leisten wollen, sind für die Feststellung der Bedeutung der Stakeholder für das Krankenhaus die Bedingungen und die Art der Zusammenarbeit zwischen Krankenhaus und Stakeholder von Interesse. Fragen zu diesen Aspekten der Stakeholder-Wichtigkeit sind beispielhaft Gegenstand des folgenden Abschnitts .

\section{Bedingungen und Art der Zusammenarbeit zwischen Krankenhaus und Stakeholder}

\section{Fragen nach den Bedingungen und der Art der} Zusammenarbeit zwischen Krankenhaus und Stakeholder

- Welcher Allokationsebene sind die Stakeholder zuzuordnen und auf welcher formalen Basis können sie ihren Einfluss geltend machen? Wie ist die Stärke der Stakeholder einzuschätzen?
- Welche kurz-, mittel- und langfristigen Ziele verfolgen die Stakeholder?

- Welche Werte und Motive stecken hinter diesen Zielen?

- Mit welchen Strategien versuchen die Stakeholder, die Ziele umzusetzen?

- Gibt es Beziehungen zwischen Stakeholdern und Verantwortungsträgern und welcher Art sind diese?

- Wie verhalten sich die verschiedenen Stakeholder zueinander? Bilden sie ihrerseits Koalitionen, um sich gegenseitig zu unterstützen, oder stehen sie eher in Konkurrenz zueinander und schwächen damit ihren Einfluss?

- Sind die Stakeholder zufrieden oder eher unzufrieden mit dem unternehmerischen Handeln der Geschäftsführung?

- ...

\section{b) Art und Stärke des Einflusses der Stakeholder auf unternehmerische Entscheidungen}

Grundlage für die Feststellung der Art und der Stärke des Einflusses, mit dem die Stakeholder die Berücksichtigung ihrer Anliegen geltend machen (können), ist deren Zuordnung zu einer der drei Allokationsebenen und zu einer der formalen Grundlagen (rechtlicher, vertraglicher, finanzieller und/oder politischer Art) für das Geltendmachen der Anliegen (s. Tab. 10).

\section{c) Ergebnis}

Anhand der bisher gesammelten Informationen lassen sich die Stakeholder einer der vier Gruppen A-D zuordnen (s. Abb. 47):

- Gruppe A: Ausgehend von der formalen Crundlage, auf der die Stakeholder ihre Anliegen geltend machen können, und deren Zuordnung zu einer der Allokationsebenen ist deren Einfluss sehr hoch. Die Beiträge der Stakeholder sind für das Krankenhaus von existenzieller Wichtigkeit. Die Geschäftsführung wird es deshalb schwer haben, den Anliegen der Stakeholder nicht zu entsprechen.

- Gruppe B: Die Stärke des Einflusses der Stakeholder ist identisch mit dem der Cruppe A. Die Stakeholder-Beiträge sind für das Krankenhaus von weniger großer Wichtigkeit.

- Gruppe C: Das Krankenhaus muss bei seinen Entscheidungen weniger Rücksicht nehmen auf eventuelle Reaktionen der Stakeholder für den Fall, dass deren Anliegen nicht ausreichend oder gar nicht berücksichtigt werden. Allerdings sind die Beiträge dieser Stakeholder für das Realisieren der Krankenhaus-Ziele von ebensolcher Wichtigkeit wie die der Beiträge der der Cruppe A zugeordneten Stakeholder. 


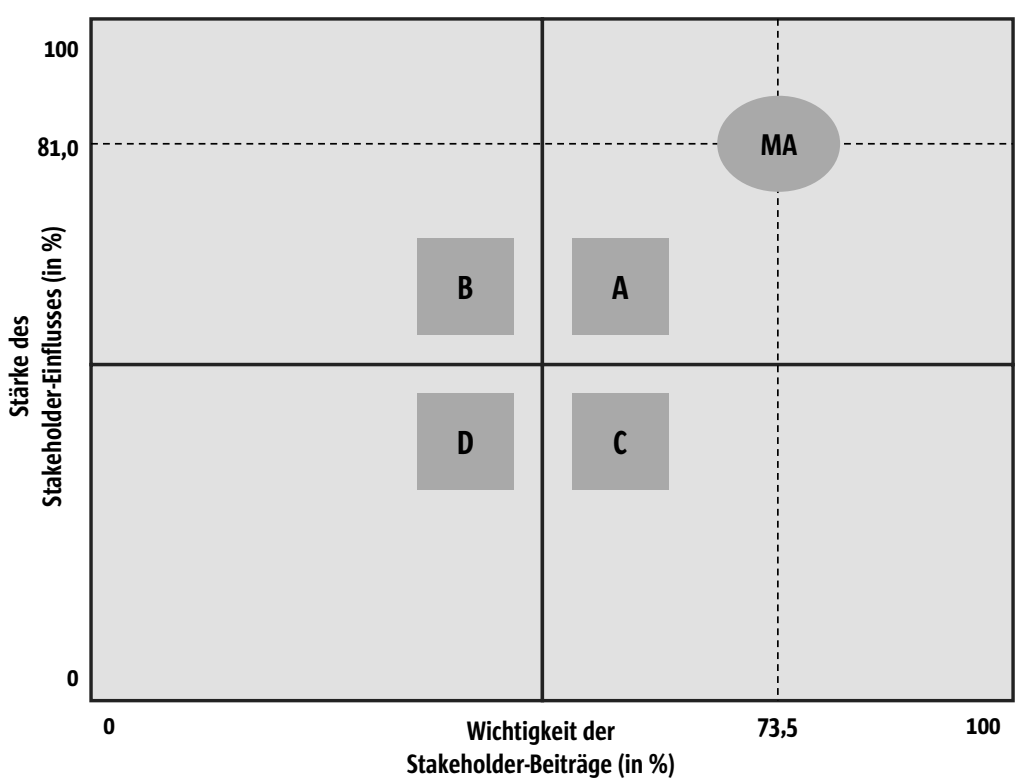

Abb. 47 Bedeutung der Stakeholder (mit Beispiel)

- Gruppe D: Die Stakeholder, die dieser Cruppe zugeordnet werden müssen, haben wenig Einfluss auf das Geschehen im Krankenhaus. Ihre Beiträge sind von weniger großer Wichtigkeit für dessen weitere Entwicklung.

Wenn ein Stakeholder der Gruppe A zugeordnet wird, verlangt die Zusammenarbeit mit diesem eine besondere Aufmerksamkeit. Die Entscheidungen, von deren Ergebnis dieser Stakeholder betroffen ist, werden sorgfältig vorbereitet. Bei nur kleinen Abweichungen von den Vorstellungen, die der Stakeholder von der Befriedigung seiner Bedürfnisse hat, muss nämlich damit gerechnet werden, dass der Stakeholder seinen starken Einfluss geltend machen wird, um eine Korrektur der Entscheidung in seinem Sinne zu erreichen. Die Beiträge, die der Stakeholder für das Krankenhaus leistet, sind zu wichtig, als dass sich das Krankenhaus eine Verschlechterung des Klimas der Zusammenarbeit leisten will. Bei einem der Cruppe D zugeordneten Stakeholder wird man dagegen - wenn er überhaupt bei unternehmerischen Entscheidungen berücksichtigt wird - weniger Aufwand in die Vorbereitung einer Entscheidung investieren, die diesen Stakeholder betrifft.

Die Zuordnung der Stakeholder zu jeweils einer der Gruppen A bis D reicht allein noch nicht aus, um entscheiden zu können, welche der in die Analyse einbezogenen Stakeholder mit ihren Anliegen bei unternehmerischen Entscheidungen berücksichtigt werden sollen. Möglicherweise sind es mehr Stakeholder mit einer hohen Bedeutung als im nächsten Analyseschritt vernünftigerweise in Betracht gezogen werden können. Es bedarf also einer Auswahl-Entscheidung und es bedarf vor allem der Regeln, anhand derer diese Entscheidung gefällt wird.

Folgende Alternativen bieten sich an:

- Eine sehr einfache Lösung dieses AuswahlProblems könnte darin bestehen festzulegen, dass entweder nur die Stakeholder berücksichtig werden, die der Gruppe A zugeordnet werden können, oder dass alle Stakeholder mit Ausnahme jener, die der Cruppe D zugeordnet werden, in Betracht kommen.

- Eine solche Lösung lässt sich deshalb vertreten, weil mit der Zuordnung der Stakeholder $z u$ einer der vier Gruppen eine Aussage über deren Bedeutung für das Krankenhaus vorliegt.

- Eine etwas differenziertere Lösung des Auswahl-Problems besteht darin, die Kennzahlen „Stärke des Stakeholder-Einflusses“ und „Wichtigkeit der Stakeholder-Beiträge“ ge- 
wichtet zu einer Gesamt-Kennzahl „Bedeutung des Stakeholders" zusammenzufassen.

\section{d) Beispiel}

Die Zuordnung eines Stakeholders in eines der Felder A - D in Abbildung 47 erfolgt nach folgendem Vorgehensmodell (dargestellt am Beispiel eines Krankenhaus-Mitarbeiters - [MA]):

\section{Beispiel}

Ein Mitarbeiter verhandelt mit seinem Vorgesetzten über die künftige Gestaltung seines Arbeitsplatzes. Bevor sich der Vorgesetzte mit seinem Mitarbeiter zu einem abschließenden Gespräch trifft, ermittelt er die Bedeutung des Mitarbeiters für den Leistungsbereich, in dem der Mitarbeiter tätig ist, und für das Krankenhaus insgesamt wie folgt:

1. Zunächst wählt der Vorgesetzte die Bewertungskriterien aus (s. Tab. 23 und 24), mit deren Hilfe die Wichtigkeit der von dem Stakeholder zu erwartenden Beiträge und die Stärke des Stakeholder-Einflusses dem Grunde nach zum Ausdruck gebracht werden.

2. Danach legt er fest, mit welchem relativen Gewicht die Bewertungskriterien bei der Ermittlung der Wichtigkeit der von dem Stakeholder zu erwartenden Beiträge und der Stärke des Stakeholder-Einflusses berücksichtigt werden sollen (Spalte 1 der Tabellen 23 und 24).

Mit dem relativen Gewicht von 45 für den Einfluss durch die „Situation auf dem Arbeitsmarkt“ (in Tab. 23) bringt derjenige, der die Bewertung vornimmt, zum Ausdruck, dass dieses Bewertungskriterium besondere Aufmerksamkeit verdient.

3. Mit den Punkten von 1 bis 10 in jeweils der Spalte 2 der Tabellen 23 und 24 wird der Stakeholder in Bezug auf die Bewertungskriterien beurteilt. Der Punkt-Wert 1 bedeutet, dass der Stakeholder das Bewertungskriterium nicht erfüllt. 10 Punkte werden dann vergeben, wenn der Stakeholder das Bewertungskriterium voll erfüllt.

Die 10 Punkte in Tabelle 23 für die „Unterstützung durch Patienten" bedeuten, dass der Einfluss des Mitarbeiters deshalb besonders hoch ist, weil die Patienten gegenüber den Verantwortungsträgern zum Ausdruck bringen, dass sie nur wegen der exzellenten Behandlung durch den Mitarbeiter dieses und nicht ein anderes Krankenhaus konsultieren.

4. Durch Multiplikation des relativen Gewichtes (Spalte 1) mit den Punkten (Spalte 2) und Addition der Produkte (Spalte 3) über alle Bewertungskriterien wird der Wert des Einflusses der Stakeholder bzw. die Wichtigkeit der Stakeholder-Beiträge ermittelt und in das Verhältnis zu der maximal erreichbaren Zahl der Punkte (= Summe der relativen Gewichte x Summe der maximal erreichbaren Zahl der Punkte $=1.000$ ) gesetzt. Der Mitarbeiter erreicht damit 81,0\% der maximal erreichbaren Stärke seines Einflusses auf die Entscheidungen seines Vorgesetzten; die Wichtigkeit seiner Beiträge werden mit 73,5\% bewertet (s. Abb. 47).

5. Die Stärke des Stakeholder-Einflusses und die Wichtigkeit der Stakeholder-Beiträge werden zu einer GesamtKennzahl „Bedeutung des Stakeholders“ (s. Tab. 25) zusammengefasst, indem die aus den Spalten 3 der Tabellen 23 und 24 übernommenen Summe Punkte (Spalte 2 in Tab. 25) mit den Relativ-Gewichten (Spalte 1 in Tab. 25) für die Stärke des Stakeholder-Einflusses (z.B. mit 60) und für die Wichtigkeit der StakeholderBeiträge (z.B. mit 40) multipliziert werden.

Tab. 23 Stärke des Stakeholder-Einflusses - am Beispiel Mitarbeiter

\begin{tabular}{|c|c|c|c|}
\hline \multirow[t]{2}{*}{ Bewertungskriterien } & relatives Gewicht & Punkte (1-10) & Summe \\
\hline & Sp. 1 & Sp. 2 & Sp. 3 = Sp. 1 x Sp. 2 \\
\hline Arbeitsvertrag & 10 & 7,5 & 75 \\
\hline Unterstützung durch Patienten & 10 & 10 & 100 \\
\hline Unterstützung durch den Vorgesetzten & 20 & 10 & 200 \\
\hline Situation auf dem Arbeitsmarkt & 45 & 8 & 360 \\
\hline Unterstützung durch Personalvertretung & 15 & 5 & 75 \\
\hline \multicolumn{4}{|l|}{$\ldots$} \\
\hline Summe & 100 & {$[40,5]$} & 810 \\
\hline
\end{tabular}


Tab. 24 Wichtigkeit der Stakeholder-Beiträge - am Beispiel Mitarbeiter

\begin{tabular}{lccc}
\hline Bewertungskriterien & relatives Gewicht & Punkte (1-10) & Summe \\
\hline Beitrag zur Sicherung der Existenz des Krankenhauses & Sp. 1 & Sp. 2 & Sp. 3 = Sp. 1 x Sp. 2 \\
\hline Ruf des Krankenhauses & 25 & 3 & 75 \\
\hline Qualität der Behandlung & 20 & 7 & 140 \\
\hline Rekrutierung von Privatpatienten & 50 & 10 & 500 \\
\hline ... & 5 & 4 & 20 \\
\hline Summe & 100 & {$[24]$} & 735 \\
\hline
\end{tabular}

\section{Ergebnis}

Der Mitarbeiter leitet seine Einfluss-Stärke (s. Tab. 23) von den Inhalten seines derzeitigen Arbeitsvertrages, von der Fürsprache der von ihm behandelten Patienten und der seiner Vorgesetzten, hauptsächlich aber von der Arbeitsmarktsituation ab. Auf Grund seines hervorragenden Rufes, der wenigen auf dem Arbeitsmarkt zur Verfügung stehenden Bewerber mit einer vergleichbaren Qualifikation und der großen Nachfrage nach diesen wird die Einfluss-Stärke mit $81 \%$ ermittelt.

Der Mitarbeiter wird vor allem wegen seines Beitrages zur Behandlungsqualität geschätzt (s. Tab. 24). Von 1000 maximal möglichen Wichtigkeits-Punkten erreicht der Mitarbeiter 735.

Der Mitarbeiter ist deshalb der Gruppe A zuzuordnen (s. Abb. 47). Er hat damit große Chancen, seine Vorstellungen durchsetzen zu können.

Die für die beiden Kennzahlen ermittelten Punkte werden zwecks des Vergleichs der Bedeutung des Mitarbeiters mit der Bedeutung anderer Mitarbeiter unter Berücksichtigung eines Gewichtes von 60 für die Kennzahl „Stärke des Stakeholder-Einflusses“ und 40 für die Kennzahl „Wichtigkeit der Stakeholder-Beiträge“ zu einer Gesamt-Kennzahl mit 78.000 Punkten zusammengefasst (s. Tab. 25).

\subsubsection{Auswahl der zu berücksichtigenden Stakeholder-Bedürfnisse}

\section{Identifizierung und Analyse der Stakeholder-Bedürfnisse}

Erst nach der Reduzierung der Stakeholder auf die für das Krankenhaus relevanten empfiehlt sich die Analyse der Stakeholder-Anliegen. Nachfolgend werden diese im Detail (beispielhaft) dargestellt.

\section{Checkliste für die Analyse der Stakeholder-Bedürfnisse (Ausschnitt)}

\section{Stakeholder/Stakeholder-Bedürfnisse}

\section{Patienten erwarten ...}

- die Leistungen des Krankenhauses zwecks Erhalten bzw. Wiederherstellen ihrer Gesundheit in der bestmöglichen Behandlungs- und Servicequalität;

- die kurzfristige Befriedigung ihrer Behandlungs- und Sicherheitsbedürfnisse; gegebenenfalls nehmen sie Fürsprecher in Anspruch, um - wenn das gewünschte Krankenhaus stark frequentiert ist - dennoch möglichst rasch aufgenommen zu werden;

Tab. 25 Gesamt-Kennzahl „Bedeutung eines Stakeholders“ - am Beispiel Mitarbeiter

\begin{tabular}{lccc} 
Kennzahlen & $\begin{array}{c}\text { relatives Gewicht } \\
\text { Punme }\end{array}$ & Bedeutung des Stakeholders \\
\hline Stärke des Stakeholder-Einflusses & Sp. 1 & Sp. 2 & Sp. 3 = Sp. 1 x Sp. 2 \\
\hline Wichtigkeit der Stakeholder & 60 & 810 & 48.600 \\
\hline Summe & 40 & 735 & 29.400 \\
\hline
\end{tabular}


- die Respektierung ihrer Individualität;

- die Berücksichtigung ihrer Wünsche und ihrer Lebensumstände;

- die Beteiligung an Entscheidungen, die Diagnostik und Therapie betreffend;

- das Einbinden in die Behandlung und in die Pflege;

- die Einweisung durch einen Haus- oder Facharzt;

- dass Ärzte, denen sie ihre Gesundheit und ihr Leben anvertrauen, das Gesundheitsproblem bestmöglich lösen und jegliche andere Interessen - wie z.B. wissenschaftlicher und/oder finanzieller Art - hintanstellen (Klemperer, S. 2100);

$\square$

\section{Niedergelassene Ärzte erwarten ..}

- im Bedarfsfall die zeitgerechte Aufnahme ihres Patienten - gegebenenfalls ohne Wartezeit - in die stationäre Behandlung;

- fortlaufende Information - gegebenenfalls durch die Teilnahme an den Visiten - über den Fortgang der Behandlung ihrer Patienten;

- zeitnah mit der Entlassung des von ihnen eingewiesenen Patienten aus der stationären Behandlung den Erhalt des Arztbriefes, der alle für die eventuell notwendige ambulante Weiterbehandlung und/oder für die Unterstützung durch einen ambulanten Pflegedienst erforderlichen Informationen enthält;

- .

\section{Mitarbeiter erwarten ...}

- die Unterstützung durch den Arbeitgeber zur Bewältigung des Konflikts, der entstehen kann, weil der Arbeitergeber einerseits einen Beitrag zur Realisierung der Unternehmensziele erwartet, der Mitarbeiter sich aber vor allem als Agent der ihm anvertrauten Patienten versteht;

- die Bereitstellung jener Ressourcen, um Patienten bestmöglich versorgen zu können;

- Arbeitsbedingungen, die es ihnen ermöglichen, ihrer Verantwortung gegenüber den Patienten und gegenüber dem Krankenhaus nachzukommen;

- Arbeitsbedingungen, die es ihnen ermöglichen, auch mit zunehmendem Alter noch die von ihnen geforderten Leistungen möglichst ohne altersbedingte Einschränkung erbringen zu können;

- möglichst dauerhaft die Befriedigung unter anderem von Sicherheits-, Zugehörigkeits-, Wertschätzungs- und Selbstverwirklichungsbedürfnissen;

- einen partizipativen Führungsstil als Grundlage für die Realisierung der genannten Ziele;

- gegebenenfalls die Unterstützung durch die Personalvertretung;
Führungskräfte $e^{62}$ erwarten ...

- die Bereitstellung eines Führungskonzepts und darin eingebettet die Führungsinstrumente, die es ihnen ermöglichen, ihrer Verantwortung gegenüber ihren Mitarbeitern nachzukommen;

- Arbeitsbedingungen, die es ihnen als leitenden Ärzten ermöglichen, Privatpatienten zu behandeln;

(1)

\section{Krankenkassen erwarten ...}

- ein möglichst hohes Maß an Behandlungs- und Servicequalität als Grundlage für die Behandlung der bei ihnen versicherten Patienten;

- im Interesse ihrer Versicherten ein befriedigendes Preis-Leistungs-Verhältnis für das Hervorbringen der Leistungen des Krankenhauses;

- ...

\section{Eigentümer erwarten ...}

- Sicherheit des eingesetzten Kapitals und dessen angemessene Verzinsung;

- finanzielle Sicherung des Krankenhauses als Grundlage für die dauerhafte Realisierung der Versorgungsund Leistungsverträge durch das Krankenhaus;

- Entwicklung von Unternehmenszielen mittel- und langfristiger Art durch die Geschäftsführung;

- Einfluss auf die Gestaltung und Realisierung der strategischen Unternehmensziele über die formal zuständigen Gremien (wie z.B. Aufsichtsrat);

- den Einsatz eines professionellen Managements;

- Implementierung eines Qualitätsmanagement- und Organisationsentwicklungs-Konzepts;

- ...

\section{Bewertung der Stakeholder-Bedürfnisse}

Wenn die Anliegen der Stakeholder identifiziert und auch genauer beschrieben worden sind, gilt es die Anliegen zu bewerten. Bewerten im Sinne einer Umweltanalyse heißt: Untersuchen, welche Chancen und Risiken sich für das Unternehmen angesichts der Befriedigung oder Nicht-Befriedigung der zu beobachtenden Bedürfnisse der Stakeholder ergeben.

Anhand eines Beispiels soll dieser Vorgang verdeutlicht werden. Dabei wird auf eines der

62 Führungskräfte sind - bis auf die Mitglieder der Geschäftsführung - immer auch Mitarbeiter. Deshalb werden hier nur jene Bedürfnisse genannt, die sie auf Grund ihrer Führungskräfte-Funktion zusätzlich zu den Mitarbeiter-Bedürfnissen geltend machen. 
im Textabschnitt Checkliste für die Analyse der Stakeholder-Bedürfnisse ausgewiesenen Bedürfnisse der Mitarbeiter abgestellt.

\section{Beispiel}

\section{Bedürfnis der Mitarbeiter}

Mitarbeiter erhoffen für sich persönlich die möglichst dauerhafte Befriedigung unter anderem von Sicherheitsbedürfnissen.

Eine genauere Analyse hat ergeben: Die Mitarbeiter verstehen unter Sicherheit unter anderem auch das sichere Bewältigenkönnen der ihnen übertragenen Aufgaben. Sie legen deshalb Wert darauf, dass die von innen zu realisierenden Ziele zwischen dem Vorgesetzten und den Mitarbeitern vereinbart werden (= Führen mit Zielvereinbarungen). Sie erwarten, dass auf der Basis einer solchen Vorgehensweise nur solche Ziele mit innen ver- einbart werden, deren Realisierung von ihnen bewältigt werden kann.

\section{Bewertung}

Die Befriedigung dieses Bedürfnisses löst Chancen und Risiken, die Nicht-Befriedigung allerdings nur Risiken aus (s. Tab. 26).

Wenn angenommen werden kann, dass sich die Befriedigung des in dem Beispiel verwendeten Mitarbeiter-Anliegens auf das Maß der Zufriedenheit der vorhandenen Mitarbeiter mit ihrer Arbeitssituation und auf das Ansehen des Krankenhauses als Arbeitgeber (= Magnet-Hospital) auswirkt, dann lässt sich dieses mittels folgender Indikatoren messen (s. Spalte 4 in Tab. 27).

Tab. 26 Bewertung der Stakeholder-Bedürfnisse (Beispiel)

\begin{tabular}{|c|c|c|}
\hline \multicolumn{3}{|c|}{ Sicherheitsbedürfnis } \\
\hline & Bedürfnis-Befriedigung & Nicht-Bedürfnis-Befriedigung \\
\hline Chancen & $\begin{array}{l}\text { Wenn die Geschäftsführung diesem Wunsch der Mit- } \\
\text { arbeiter nachkommen will, erhöht dieses deren Ar- } \\
\text { beits-Zufriedenheit mit der Folge, dass die Qualität } \\
\text { und die Effizienz der Leistungserbringung und damit } \\
\text { auch die finanzielle Situation des Krankenhauses ver- } \\
\text { bessert werden (können). }\end{array}$ & $\ldots$ \\
\hline & $\begin{array}{l}\text { Das Krankenhaus entwickelt das Führen mit Zielver- } \\
\text { einbarungen zu einem Alleinstellungsmerkmal. Als } \\
\text { Magnet-Hospital wirkt es auf Patienten wie auf poten- } \\
\text { zielle Mitarbeiter gleichermaßen anziehend. Vorhan- } \\
\text { dene Mitarbeiter lassen sich an das Krankenhaus bin- } \\
\text { den; das Rekrutieren neuer Mitarbeiter wird unter- } \\
\text { stützt. }\end{array}$ & \\
\hline Risiken & $\begin{array}{l}\text { Führen mittels Zielvereinbarungen setzt voraus, dass } \\
\text { die Führungskräfte dafür gewonnen werden können, } \\
\text { mit diesem Instrument zu arbeiten. Die Vorgesetzten } \\
\text { werden gegebenenfalls in die Lage versetzt, dieses } \\
\text { Instrument sachgerecht anzuwenden. } \\
\text { Wenn diese Voraussetzungen nicht realisiert werden } \\
\text { können und die eine oder andere Führungskraft nur } \\
\text { so tut als ob und damit die guten Absichten der Ge- } \\
\text { schäftsführung konterkariert, wird der Ruf des Kran- } \\
\text { kenhauses als Arbeitgeber eher schlechter als wenn } \\
\text { man es bei dem bisherigen Zustand belassen hät- } \\
\text { te; das Risiko, qualifizierte Mitarbeiter nicht mehr } \\
\text { in ausreichender Zahl rekrutieren zu können, steigt. }\end{array}$ & $\begin{array}{l}\text { Wenn das Krankenhaus darauf verzichtet, ein zeit- } \\
\text { gemäßes Führungskonzept zu realisieren, das unter } \\
\text { anderem das Führen mit Zielvereinbarungen als } \\
\text { Führungsinstrument vorsieht, dann muss es ange- } \\
\text { sichts der sich abzeichnenden Arbeitsmarkt-Entwick- } \\
\text { lungen damit rechnen, ausreichend qualifizierte und } \\
\text { motivierte Mitarbeiter nicht mehr in bedarfsgerech- } \\
\text { ter Zahl rekrutieren zu können. }\end{array}$ \\
\hline
\end{tabular}


Tab. 27 Beispiel für das Messen der Folgen der Befriedigung eines Mitarbeiter-Bedürfnisses (Ausschnitt)

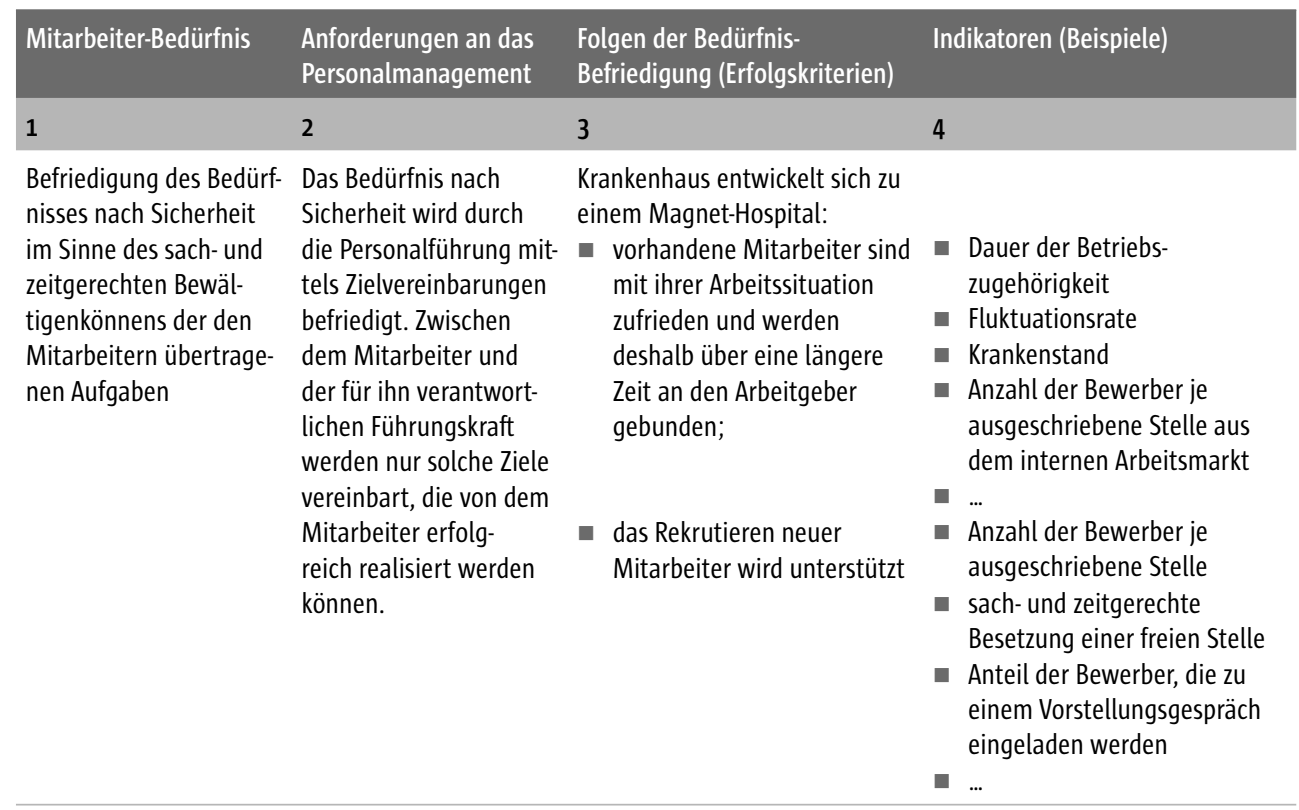

\subsubsection{Zusammenfassung}

Die Umweltanalyse auf Stakeholder-Basis wird in zwei Schritten durchgeführt:

1. Aus der großen Zahl von Stakeholdern werden diejenigen ausgewählt, deren Bedürfnisse bei unternehmerischen Entscheidungen der Krankenhaus-Verantwortungsträger - soweit diese als legitim akzeptiert werden - berücksichtigt werden sollen. Als Auswahlkriterien kommt einerseits die Wichtigkeit der von den Stakeholdern zu erwartenden Beiträge für die Realisierung der Unternehmensziele in Betracht. Zum zweiten sind es die Art und die Stärke des Einflusses, den Stakeholder geltend machen können, um zu erreichen, dass ihre Bedürfnisse bei Entscheidungen der Krankenhaus-Verantwortungsträger angemessen berücksichtigt werden.

Die hier vorgestellte Methode gestattet es, die ausgewählten Stakeholder hinsichtlich ihrer Bedeutung für das Krankenhaus zu vergleichen. Unter anderem lässt sich aus diesem Vergleich ableiten, mit welchem Gewicht die Stakeholder-Bedürfnisse bei Entscheidungen berücksichtigt werden.

2. Die Bedürfnisse der ausgewählten Stakeholder werden identifiziert und bewertet, indem die
Chancen und Risiken dargestellt werden, die sich für das Krankenhaus im Falle der Befriedigung bzw. der Nicht-Befriedigung der Stakeholder-Anliegen ergeben. Anhand des Bewertungsergebnisses kann entschieden werden, welche Bedürfnisse mit welchem Gewicht - dieses ergibt sich allerdings erst aus dem Vergleich der Ergebnisse der Stakeholder-Bedürfnis-Bewertungen - bei Entscheidungen berücksichtigt werden.

Mit der Präsentation eines Vorgehensmodells für die Umweltanalyse auf Stakeholder-Basis wird deutlich, dass die besondere Stärke der verwendeten Analyse-Instrumente darin zu sehen ist, dass sie

- eine gute, weil Transparenz schaffende Basis für den Dialog zwischen den Verantwortungsträgern und den von deren Entscheidungen betroffenen und

- detaillierte Begründungen für die zu fällenden Entscheidungen liefern.

Was die vorgestellten Analyse-Instrumente aber nicht leisten können: Sie können den Dialog zwischen den erwähnten Partnern nicht ersetzen. 


\subsection{Unternehmensanalyse}

\subsubsection{Einführung}

In der Umweltanalyse auf Stakeholder-Basis geht es darum festzustellen, welche für das Krankenhaus relevanten Stakeholder es gibt, was diese Stakeholder von dem Krankenhaus erwarten, mit welchen Beiträgen diese sich an der Realisierung der Ziele des Krankenhauses beteiligen wollen und welchen Einfluss sie auf das Handeln im Krankenhaus ausüben können. Das Ergebnis der Unternehmensanalyse zeigt dagegen die Stärken und die Schwächen des Unternehmens in Bezug auf die Wahrnehmung von Verantwortung. Es gibt Antworten auf die Fragen,

- wie wichtig den Verantwortungsträgern die Berücksichtigung von Stakeholder-Bedürfnissen bei ihren Entscheidungen ist und

- wie die institutionellen und personellen Voraussetzungen für sozial verantwortliches Handeln in dem Krankenhaus beschaffen sind.

\subsubsection{Berücksichtigung der Stakeholder-Anliegen bei unternehmerischen Entscheidungen}

Der nächste Schritt der Standortbestimmung besteht also in der Beantwortung der Frage, ob überhaupt und mit welchem Gewicht die Verantwortungsträger die Bedürfnisse der Stakeholder bei Entscheidungen berücksichtigen. In dem Fragebogen in nachfolgendem Abschnitt werden (in Anlehnung unter anderem an Göbel 1992, S. 21off.; Paul Gerhardt Diakonie e.V., S. 5 ff.; DRK Kliniken Berlin, S. 7 ff.) beispielhaft Fragen gestellt, deren rückhaltlose Beantwortung Aufschluss über das Verständnis der Verantwortungsträger im Krankenhaus zu sozial verantwortlichem Handeln und über den Umgang des Krankenhauses mit den Stakeholder-Anliegen gibt. In den Fragebogen werden StakeholderAnliegen einbezogen, wie sie an mehreren anderen Stellen dieser Untersuchung - unter anderem im Textabschnitt Checkliste für die Analyse der Stakeholder-Bedürfnisse (Ausschnitt) - schon erörtert worden sind.

Die Anliegen einzelner Stakeholder sind möglicherweise sehr zahlreich und vielfältig, so dass die Beantwortung der erwähnten Fragen - unabhängig davon, wie diese organisiert wird - ein Unternehmen längere Zeit lahm legen kann. Es empfiehlt sich deshalb, die Bedürfnisse dieser
Stakeholder jeweils in Bedürfnis-Cruppen zusammen zu fassen und die Bedürfnis-Gruppen nach Wichtigkeiten zu ordnen; die Stärken-Schwächen-Analyse für die Bedürfnis-Cruppen wird entsprechend der Wichtigkeitsverteilung auf einer Zeitachse angeordnet.

Dem im Folgenden dargestellten Fragebogen wird beispielhaft und nicht für alle bisher jeweils in die Betrachtungen einbezogenen Stakeholder eine solche Gruppierung zu Grunde gelegt.

\section{Checkliste für die Unternehmensanalyse (Ausschnitt)}

\section{Stakeholder/Stakeholder-Bedürfnis-Gruppen/ Fragen zu deren Befriedigung}

Patient

\section{Zugang zu Gesundheitsdienstleistungen}

(in qualitativer, zeitlicher und örtlicher Hinsicht)

- Bieten wir die in unserem Einzugsgebiet von vielen Menschen nachgefragten Leistungen an?

- Stellen wir sicher, dass Patienten uneingeschränkt Zugang in unser Krankenhaus haben und qualifiziert untersucht werden können?

- Gibt es Vereinbarungen mit anderen Krankenhäusern, damit sichergestellt ist, dass die Patienten, die bei uns nicht behandelt werden können, von dort unverzüglich übernommen werden können?

- Stellen wir sicher, dass die Behandlung der Patienten nach dem jeweils neuesten Stand der medizinischen Forschung erfolgt?

- Werden Patienten, mit denen ein Behandlungstermin vereinbart worden ist, entsprechend dem vereinbarten Termin und ohne Wartezeit behandelt?

-

\section{Behandlungsqualität}

- Erfolgt bei uns die Behandlung auf der Grundlage ebmgestützter Behandlungsleitlinien?

- Verfügen wir über ein leistungsfähiges Fehlermanagement zwecks Minimierung der Behandlungsrisiken?

- Werden Beschwerden unverzüglich bearbeitet?

- Werden die Patienten über die Art ihrer Erkrankung und die vom Arzt geplante Behandlung und deren Erfolgsaussichten ausführlich aufgeklärt?

$\square$

\section{Zuwendung}

- Sprechen wir den Patienten als unverwechselbare Persönlichkeit mit Körper, Seele und Geist an?

- Ist menschliche Zuwendung unter Beachtung der Würde des Menschen für uns selbstverständlich? 
- Respektieren wir die Würde und die Individualität unserer Patienten unabhängig von Herkunft, Religion und sozialem Status?

- Ist unser Verhalten gegenüber den Patienten frei von sachfremden Interessenverflechtungen und Abhängigkeiten?

- ...

\section{Ethikkomitee (Steinkamp; Gordijn, S. 175)}

- Haben wir ein Forum zur sozialen Auseinandersetzung mit Fragen zu der Patienten-Behandlung?

- Ist gewährleistet, dass soziale Empfehlungen als Grundlagen von Leitlinien entwickelt und durch die dafür zuständigen Gremien verbindlich gemacht werden können?

- Sind die Voraussetzungen für soziale Fallbesprechungen geschaffen?

-..

\section{Mitarbeiter (Oldham; Janson; Purdy, S. 57-71)}

\section{Mitarbeiter als Agent der Patienten}

- Ist sichergestellt, dass Mitarbeiter uneingeschränkt loyal gegenüber den Patienten sein können?

- Ist gewährleistet, dass Mitarbeiter allein im Interesse der Patienten handeln können?

- Lassen die Arbeitsbedingungen es zu, dass die Mitarbeiter die Patienten nach ebm-gestützten Leitlinien behandeln können?

- ...

\section{Selbstverwirklichungsbedürfnis}

- Ist der Arbeitsplatz so gestaltet, dass er die Eigenverantwortung der Mitarbeiter stärkt?

- Ist gewährleistet, dass der Mitarbeiter ein abgeschlossenes Arbeitsstück abliefern kann?

- Ist der Arbeitsplatz so gestaltet, dass ein bedeutsamer Nutzen für andere innerhalb und/oder außerhalb des Unternehmens dem Inhaber des Arbeitsplatzes eindeutig zugeordnet werden kann?

- Unterstützen wir mit unserer Personalentwicklung die qualifizierte Aufgabenwahrnehmung, den systematischen Ausbau der Fähigkeiten unserer Mitarbeiter und deren Identifikation mit ihren Aufgaben und mit unserem Unternehmen?

-

\section{Wertschätzungsbedürfnisse}

- Wird geleistete Arbeit durch die Vorgesetzten anerkannt?

- Werden unsere Mitarbeiter zunächst als „Lösungsfaktor" und weniger als „Kostenfaktor" gesehen und behandelt?
- Werden die Mitarbeiter von ihren Vorgesetzten im Sinne eines partizipativen Führungsstils in das Vorbereiten und das Fällen von Entscheidungen einbezogen?

- Besteht für die Mitarbeiter die Chance, im Unternehmen Karriere zu machen?

- Werden die Mitarbeiter bei der Wahrnehmung ihrer Karriere-Chancen außerhalb des Krankenhauses unterstützt?

- Erhalten die Mitarbeiter eine Information über das Ergebnis ihrer Arbeit?

- Werden die Mitarbeiter über alle betrieblichen Vorgänge ausreichend und zeitnah unterrichtet?

- ...

\section{Soziale Bedürfnisse}

- Werden unsere Führungskräfte ihrer Vorbildfunktion gerecht?

- Wird der Wunsch unserer Mitarbeiter, Mitglied einer Gruppe (wie z.B. einer Station, einer Abteilung, des Krankenhauses) zu sein, erfüllt?

- Werden unsere Mitarbeiter in ihrer Entwicklung gefördert?

- Können sich unsere Mitarbeiter auf ihre Vorgesetzte/ Kollegen verlassen?

- Werden Probleme offen angesprochen?

- Werden Fehler eher personalisiert oder vor allem als Lernchance begriffen?

- ...

\section{Sicherheitsbedürfnisse}

- Ist die Personalpolitik auf eine Sicherung der Arbeitsplätze ausgerichtet?

- Ist der Arbeitsplatz gesundheitsfördernd gestaltet?

- Sind die Löhne und Gehälter leistungsgerecht und sichern sie die Finanzierung einer angemessenen Lebensführung?

- Ist der Arbeitsplatz so gestaltet, dass unsere Mitarbeiter die mit innen vereinbarten Ziele im Regelfall erreichen können?

- ...

\section{Führungskräfte}

\section{Führungskonzept}

- Ist sichergestellt, dass das Unternehmen über eine zeitgemäße und die spezifischen kulturellen und Produktionsbedingungen des Krankenhauses berücksichtigende Führungsorganisation verfügt?

- Stehen den Führungskräften zeitgemäße Instrumente der Personalführung zur Verfügung?

- Werden Personalführungsinstrumente - wie z.B. „Führen mit Zielvereinbarungen“ und „Mitarbeiterorientierungsgespräch“ - genutzt? 
- Werden den Führungskräften erforderlichenfalls Hilfen angeboten, damit sie ihre Führungskompetenz ausbauen können?

口

\section{Führungsgrundsätze}

- Gibt es einen Katalog von Führungsgrundsätzen, die unter Beteiligung der Mitarbeiter entwickelt worden sind?

- Sind die Führungsgrundsätze unter Berücksichtigung der Grundpostulate des Managements der sozialen Verantwortung erarbeitet worden?

- ...

\section{Eigentümer}

Sicherung des zur Verfügung gestellten Kapitals

- Gehen wir unkalkulierbare Risiken ein?

- Verwenden wir die Gewinne der Erlöse über die Kosten gemäß der betrieblichen Satzung?

- Setzen wir das Kapital sicher und Ertrag bringend ein?

- Haben wir ein Konzept des Risk Managements und setzen wir dieses um?

- ...

\section{Führungskonzept (Eiff, v., S. 66)}

- Konnten wir den Eigentümer davon überzeugen, dass wir ein geeignetes Führungskonzept haben und dass dieses gemäß den sich ändernden internen und externen Herausforderungen so weiterentwickelt wird, dass den Interessen des Eigentümers entsprochen werden kann?

- Ist das Führungskonzept auf unsere Unternehmenskultur zugeschnitten und steht die realisierte Führungstechnik und das gelebte Führungsverhalten im Einklang mit unserem Führungskonzept, sodass das Umsetzen der Unternehmensziele gewährleistet werden kann?

- ...

\section{Qualifikation der Geschäftsführung}

- Gibt es Programme zur weiteren Professionalisierung unseres Managements als Voraussetzung für die Wahrnehmung komplexer Führungsaufgaben?

Die Beantwortung der Fragen wird möglicherweise einen mehr oder weniger umfangreichen Maßnahmen-Katalog zur Folge haben. Dessen Abarbeitung wird - je nachdem, welche Ressourcen zur Erledigung dieser Aufgabe bereit gestellt werden können - einen mehr oder weniger langen Zeitraum in Anspruch nehmen. Es empfiehlt sich deshalb, die Maßnahmen mit den Betroffenen gemeinsam zu priorisieren (s. Grundpostulate: „Berücksichtigung der Interessen Betroffener“, „Förderung der argumentativen, dialogischen Verständigung“ und eventuell anderer), um möglichst rasch die ersten Ergebnisse präsentieren und damit das Konzept des Managements der sozialen Verantwortung festigen zu können.

\subsubsection{Eignung der organisatorischen Grundstruktur?}

Die Beantwortung der Fragen im Abschnitt Checkliste für die Unternehmensanalyse (Ausschnitt) lässt noch nicht erkennen, welches die Cründe für die möglicherweise nicht sachgerechte Berücksichtigung der Stakeholder-Anliegen bei unternehmerischen Entscheidungen sind. Dazu bedarf es weiterer Analysen. Die erste davon geht der Frage nach, ob die strukturellen Voraussetzungen gegeben sind, um die Geschäftsführung sowie alle übrigen Verantwortungsträger dabei $\mathrm{zu}$ unterstützen, die ihnen zugewiesene Verantwortung erfolgreich wahrnehmen zu können. Dabei werden in den weiteren Ausführungen unter Unternehmensstruktur die Leitungsstruktur des Krankenhauses und der Prozess der betrieblichen Leitungsentscheidung verstanden.

Die Anforderungen an die Leitungsstruktur des Krankenhauses und an die Gestaltung des Prozesses betrieblicher Leitungsentscheidungen werden in den Kapiteln 6.4, 6.5 und 7.4.2 ausführlich, wenn auch nur beispielhaft, dargestellt. Bei der Prüfung, ob die gegebene organisatorische Grundstruktur des Krankenhauses geeignet ist, sozial verantwortliches Handeln zu unterstützen oder ob es organisationale Barrieren gibt, die die Umsetzung von Verantwortung behindern, kann auf die dort ausgewiesenen Normen zurück gegriffen werden. Auf eine erneute Darstellung in diesem Kapitel kann verzichtet werden.

\subsubsection{Eignung der Verantwortungsträger?}

Ein weiterer Grund für das unzureichende Umsetzen der Goldenen Regel kann darin bestehen, dass die Verantwortungsträger entweder nicht wissen, was es heißt, Verantwortung wahrzunehmen, oder dass sie von der Nützlichkeit - auch für sich selbst -, sozial verantwortlich zu handeln, nicht überzeugt sind. Die Führungskräfte sind deshalb 
aufgerufen, sich von den Fähigkeiten und von der Einstellung ihrer Mitarbeiter, das Wahrnehmen von Verantwortung betreffend, zu überzeugen. Ein Ansatz zur Analyse der eventuell vorhandenen Fähigkeits- und/oder Wollen-Defizite besteht in dem Vergleich des für Verantwortungsträger relevanten Anforderungsprofils (s. Tab. 19) mit deren Fähigkeiten (s. Abb. 48).

Wenn die Führungskräfte feststellen, dass ihre Mitarbeiter Fähigkeits-, möglicherweise auch Wollen-Defizite hinsichtlich des Wahrnehmens von Verantwortung haben, schließen sich analog zu der Mehrstufigkeit des Controllings zwei Maßnahmen an:

- Zu der Analyse der Cründe für das unzureichende Wahrnehmen von Verantwortung zählt die Überprüfung der bisher verwendeten Personalbeschaffungs-, Personalentwicklungs- und Personalführungsprogramme sowie die der geltenden Anreizsysteme (in Anlehnung Kreikebaum, S. 277) daraufhin, ob sie geeignet sind, personelle Barrieren, die der Umsetzung des Konzepts des Managements der sozialen Verantwortung im Wege stehen, zu überwinden. Die Anforderungen, die an eine das Wollen und das Können des Realisie- rens des neuen Unternehmensleitbildes unterstützende Personalarbeit zu stellen sind und anhand derer analysiert werden kann, ob die personellen Voraussetzungen für das Realisieren des Managements der sozialen Verantwortung gegeben sind, werden in den Kapiteln 7.2 und 7.3 und vor allem im Kapitel 6.4.3, Personalarbeit, im Detail, aber nicht mit dem Anspruch auf Vollständigkeit, beschrieben. Auf diese Normen kann zum Zwecke der Prüfung der Eignung der Verantwortungsträger und der Analyse einer eventuell festgestellten unzureichenden Eignung zurückgegriffen werden.

- Wenn festgestellt wird, dass die Verantwortungsträger nicht entsprechend der Goldenen Regel handeln, muss auch danach gefragt werden, ob Veränderungen in der Handlungssituation des Krankenhausmanagements zu diesem Defizit beigetragen haben.

\section{Beispiel}

Es gibt in der jüngsten Vergangenheit immer mehr Krankenhäuser, die vakante Stellen für Ärzte nicht oder nicht zeitgerecht und qualifiziert besetzen können. Die in die-

\begin{tabular}{|c|c|c|c|c|c|c|c|}
\hline \multirow{2}{*}{$\begin{array}{l}\text { Fähigkeitsmerkmale } \\
\text { Ausschnitt: soziale Fähigkeiten }\end{array}$} & \multicolumn{7}{|c|}{ Merkmalsausprägung } \\
\hline & 1 & 2 & 3 & 4 & 5 & 6 & 7 \\
\hline $\begin{array}{l}\text { - Dialogfähigkeit } \\
\text { - } \text { abweichende Meinungen und Ansichten } \\
\text { respektieren } \\
\text { - Kooperationsvermögen } \\
\text { - Verhalten, das durch Achtung gegenüber den } \\
\text { Bedürnissen anderer und durch Solidarität } \\
\text { gekennzeichnet ist } \\
\text { - Toleranz } \\
\text { - Konsenswillen } \\
\text { - Ehrlichkeit } \\
\text { - Offenheit } \\
\text { - wechselseitige Anerkennung } \\
\text { - Verantwortungsbewusstsein } \\
\text { - Einfühlungsvermögen } \\
\text { - Bereitschaft, an der Entwicklung des } \\
\text { Unternehmens mitzuwirken }\end{array}$ & & & & & & & \\
\hline $\begin{array}{l}1=\text { niedrig ausgeprägt } \\
7=\text { hoch ausgeprägt }\end{array}$ & \multicolumn{7}{|c|}{$\begin{array}{ll} & \text { Fähigkeitsprofil } \\
-\ldots-\ldots-1 & \text { Anforderungsprofil }\end{array}$} \\
\hline
\end{tabular}

Abb. 48 Fähigkeitsprofil eines Verantwortungsträgers (Ausschnitt) 
sen Krankenhäusern für die Patientenbehandlung verantwortlichen können deshalb die Bedürfnisse der Patienten und/oder die der Mitarbeiter möglicherweise nur eingeschränkt befriedigen.

Ausgehend von den festgestellten Fähigkeitsund Wollen-Defizite werden - ein ausreichendes Entwicklungspotenzial vorausgesetzt - geeignete Entwicklungsmaßnahmen geplant und realisiert (Näheres zu den Zielen und Instrumenten der Einstellungsentwicklung und der Fähigkeitsentwicklung s. Kap. 7.3.3 und 7.4.1).

\subsection{Verantwortung konkretisieren}

Damit das Umsetzen des Managements der sozialen Verantwortung sich nicht in - häufig nicht sehr wirkungsvollen - Appellen an das Verantwortungsbewusstsein der Verantwortungsträger erschöpft und die Basis zur Verfügung steht, mittels derer das Realisieren des neuen Management-Konzepts evaluiert werden kann (Oppenrieder, S. 2), werden Normen benötigt, anhand derer sozial verantwortliches Handeln sich ausrichtet (Berkel; Herzog, S. 88).

Mit „Verantwortung konkretisieren“ wird der Prozess verstanden, der zu diesen Normen führt. Dieser Prozess wird auf zwei Ebenen realisiert:

1. Das Ergebnis des Normenfindungsprozesses ist das Unternehmensleitbild. Mit den dort ausgewiesenen Normen teilt die Geschäftsführung den Verantwortungsträgern im Krankenhaus mit, welche Handlungen erlaubt/ erwünscht bzw. verboten/unerwünscht sind. Das Unternehmensleitbild basiert auf dem in dem Krankenhaus gelebten Wertesystem, also auf den Einstellungen, die die Verantwortungsträger zum Beispiel hinsichtlich des Umgangs der Beschäftigten im Krankenhaus untereinander sowie zu den Patienten und deren Angehörigen und zu anderen Stakeholdern für wünschenswert halten.

Der Prozess der Formulierung des Unternehmensleitbildes, die Entwicklung der Normen also, die das Handeln der Verantwortungsträger in einem Krankenhaus leiten, erfolgt unter Berücksichtigung von Kriterien und Regeln, die unter dem Begriff „Grundpostulate“ zusammengefasst werden.

2. Die Konkretisierung der Verantwortung setzt deshalb zunächst die Entwicklung der Grundpostulate des Managements der sozialen Ver- antwortung und des Werte- und Normensystems voraus.

Damit die Grundpostulate und das Wertesystem die Gunst derer finden können, deren Handeln durch sie geleitet werden soll, bietet es sich an, sie unter Verwendung einiger der Grundpostulate gewissermaßen als Mega-Regeln zu entwickeln:

1. So sollten die Interessen derer, deren Handeln durch die Normen des Unternehmensleitbildes und damit mittelbar auch durch die Grundpostulate maßgeblich bestimmt wird, in angemessener Weise berücksichtigt werden.

2. Sie sollten das Ergebnis einer argumentativen, dialogischen Verständigung mit den Betroffenen gemäß Ziffer 1. sein.

3. Die Geschäftsführung sollte als Grundlage für die Entwicklung der Grundpostulate und des Wertesystems ein Arbeitsklima schaffen, das die Mündigkeit der an der Entwicklung beteiligten fördert.

4. Nicht nur der Text der Grundpostulate und des Wertesystems, sondern auch deren Begründung sollten von allen, die an dem Entstehen der Grundpostulate beteiligt waren, einvernehmlich getragen werden.

Soweit das methodische Vorgehen im Überblick. Damit der angestrebte Konsens als rational im Sinne von einvernehmlich qualifiziert werden kann und als Ergebnis Verantwortung konkret und fassbar ist, muss die Argumentation der an dem Prozess beteiligten einige formale und inhaltliche Bedingungen erfüllen (Berkel; Herzog,

S. 104f. und die dort genannte Literatur):

1. Die Diskussionsteilnehmer müssen bereit sein, ihre Meinung und Anliegen in Frage stellen zu lassen.

2. Sie müssen auf Appelle an nicht überprüfte Werte und Prinzipien verzichten.

3. Ebenso müssen die Diskussionsteilnehmer auf Sanktionen für die Zustimmung oder Ablehnung ihrer Argumentation verzichten.

4. Sie müssen imstande sein, gute Gründe, die Zustimmung erwarten lassen, vorzutragen.

\subsection{Für Verantwortungsbewusstsein sensibilisieren}

Es wäre im Regelfall falsch anzunehmen, die neuen Unternehmensgrundsätze würden von allen 
Akteuren des Krankenhauses begierig aufgenommen und dauerhaft umgesetzt. Im Gegenteil: Die Geschäftsführung sowie die Führungskräfte müssen sich darauf einstellen, dass es im Einzelfall großer Anstrengungen bedarf, um die Mitarbeiter von der Notwendigkeit und der Zweckmäßigkeit des neuen Unternehmensleitbildes überzeugen zu können.

Diese Aussage steht im Übrigen nicht im Widerspruch zu der Annahme an anderer Stelle, die Mitarbeiter würden gerne sozial verantwortlich handeln, es fehle ihnen nur die einschlägige Unterstützung. Ein neues Unternehmensleitbild wird, auch wenn es dem skizzierten Wunsch der Mitarbeiter entgegenkommt, dennoch zunächst als Einengung gefühlt und deshalb mit Skepsis aufgenommen. Das liegt vor allem an der Forderung der Geschäftsführung, die Werte und Normen des Unternehmensleitbildes konsequent und ohne Ausnahme zur Grundlage des täglichen Arbeitshandelns zu machen.

Selbst wenn es der Geschäftsführung gelingt, die institutionellen Voraussetzungen für das Praktizieren der Goldenen Regel zu schaffen, wenn die Mitarbeiter wissen, was von ihnen erwartet wird, und wenn diese notwendigenfalls einschlägig weitergebildet worden sind: Wenn es die Geschäftsführung nicht schafft, die Bereitschaft der Verantwortungsträger für verantwortungsbewusstes Handeln zu wecken, muss die Überleitung des bisher praktizierten, konventionellen in das bessere Management-Konzept scheitern. Weil die Lösung dieses Problems von so zentraler Bedeutung ist, soll dieses Thema - obwohl es bereits ausführlich in Kapitel 7.3 erörtert worden ist - noch einmal mit einigen zusätzlichen Aspekten aufgegriffen werden.

Das Sensibilisieren der Verantwortungsträger in allen Hierarchieebenen und Leistungsbereichen des Krankenhauses für sozial verantwortliches Handeln erfolgt auf drei Ebenen (in Anlehnung an Göbel 2006, S. 225):

- Die Geschäftsführung sowie die Führungskräften müssen lernen und akzeptieren, dass die Steuerungswirkung des Rechts und die Mechanismen des Marktes allein nicht ausreichend sind, um die als legitim akzeptierten Bedürfnisse der Stakeholder befriedigen zu können und um Konflikte zwischen Stakeholdern mit konkurrierenden Bedürfnissen zu lösen.

- Die Führungskräfte und die Mitarbeiter müssen lernen, dass es nicht genügt, ihre Ent- scheidungen auf der Crundlage einschlägiger rechtlicher Vorschriften zu fällen; dieses wird als Minimum von der Geschäftsführung erwartet. Die Grundpostulate des Managements der sozialen Verantwortung sind daneben wichtiger Bestandteil für das Vorbereiten und das Fällen sowie für das daran sich anschließende Umsetzen einer Entscheidung. Es muss die Bereitschaft der Führungskräfte und der Mitarbeiter entwickelt werden, die Crundidee der Goldenen Regel zu akzeptieren und anzuwenden.

- Den Führungskräften und den Mitarbeitern muss das Wissen vermittelt werden, zu erkennen, welche Folgen unternehmerische Entscheidungen haben können. Erforderlich sind Weitsicht und Voraussicht, also die Fähigkeit, die in naher oder fernerer Zukunft möglicherweise eintretenden Folgen abschätzen zu können.

\section{Für Verantwortungsbewusstsein sensibilisieren heißt also, die Verantwortungsträger dafür ge- winnen, dass diese die innen zur Verfügung ste- henden Freiheiten im Sinne der Goldenen Regel nutzen.}

Nicht selten sind es Konflikte unterschiedlicher Art, die das Befassen mit dem Konzept des Managements der sozialen Verantwortung auslösen (Berkel; Herzog, S. 79f.; Kreikebaum, S. 22of.). Gemeinsam ist ihnen, dass ein Verantwortungsträger bei seinen Entscheidungen die Bedürfnisse eines Verantwortungs-Adressaten und die Folgen für den Betroffenen nicht angemessen berücksichtigt hat.

Die Identifizierung und die sorgfältige Analyse der Konflikte, die die Krankenhausarbeit begleiten, lassen erkennen, welche Stakeholder-Anliegen nicht angemessen berücksichtigt worden sind und welches die Gründe waren, die zu dem Entstehen der Konflikte beigetragen haben. Die Konflikt-Analyse liefert somit die Argumente, die von der Geschäftsführung benötigt werden, um die Krankenhaus-Akteure für Verantwortungsbewusstsein sensibilisieren und um deren Bereitschaft entwickeln zu können für das Begehen eines Weges, der viel Kraft kosten wird und der mit der Implementierung des besseren Management-Konzepts endet.

Im Folgenden werden einige Beispiele von Konfliktfeldern dargestellt. Dabei handelt es sich 
um Konflikte, die anhand der Beantwortung der in der Checkliste für die Unternehmensanalyse (Ausschnitt) aufgelisteten Fragen deutlich werden könnten.

Beispiele von Konflikten (in Anlehnung an Dahm 1993, S. 6f.; Dahm 1994, S. 4 und Berkel; Herzog, S. 81)

\section{Adressat der Verantwortung/Konflikt}

\section{Patient}

- Die Unkenntnis der Patienten wird durch die Mitglieder des therapeutischen Teams unredlich ausgenutzt.

- Patienten werden Ressourcen vorenthalten.

- Es wird mit Methoden behandelt, deren Wirksamkeit noch nicht hinreichend getestet worden sind.

- Obwohl die stationäre Behandlung aus medizinischer Sicht angezeigt ist und freie Kapazitäten - quantitativer und qualitativer Art - vorhanden sind, werden $\mathrm{Pa}$ tienten nicht aufgenommen, sondern an ein anderes Krankenhaus verwiesen.

-

\section{Mitarbeiter}

- fehlende Glaubwürdigkeit der Führungskraft - Auseinanderfallen von Sagen und Tun,

- Missachtung der Mitarbeiter durch die Führungskraft,

- messen mit zweierlei Maß: die Führungskraft reklamiert für sich selbst das Recht auf die Befriedigung diverser Bedürfnisse, von den Mitarbeitern wird das Erfüllen von Pflichten eingefordert,

- für eine Kündigung werden Gründe konstruiert,

- im Falle einer betriebsbedingten Kündigung werden die Regeln für die Zuweisung von Mitarbeitern in den Kreis der zu kündigenden Mitarbeiter (= Sozialauswahl) mit einem hohen Maß an Willkür angewendet,

- die Personalausstattung der einzelnen Leistungsbereiche eines Krankenhauses ist das Ergebnis einer Topdown-Planung und für die Mitarbeiter nicht nachvollziehbar,

- ...

\section{Eigentümer}

- Unter Hinweis auf den Grundsatz der Vorsicht (\$ 252 Abs. 1 Nr. 4 HGB) werden durch überhöhte Abschreibungen stille Reserven gebildet, die jedoch weder im Interesse der Gläubiger noch (wegen des damit verbundenen geringeren Gewinnausweises) der Eigentümer des Unternehmens sind.

-

\subsection{Konzept des Managements der sozialen Verantwortung festigen}

\subsubsection{Einführung}

Abschließend geht es um die Beantwortung der Frage, was die Geschäftsführung tun kann, damit sozial verantwortliches Handeln dauerhaft zum Bestandteil des Krankenhaus-Alltags wird. Dafür stehen ihr mehrere Instrumente zur Verfügung, von denen einige in anderen Kapiteln dieses Buches bereits Thema waren. Dabei handelt es sich um die Realisierung einer angemessenen Unternehmensstruktur sowie um die Formulierung und die Diffusion eines Unternehmensleitbildes.

Zwei weitere, der Festigung des neuen Management-Konzepts dienende Instrumente sollen in den nächsten Kapiteln zum Thema gemacht werden:

1. Der Umgang mit Verantwortung wird zum Bereich und Gegenstand des Controllings gemacht (s. Kap. 8.7.2).

2. Einzelne Teile des neuen ManagementKonzepts werden kurzfristig umgesetzt (s. Kap. 8.7.3)

\subsubsection{Wahrnehmung von Verantwortung als Gegenstand des Controllings}

Das Konzept des Managements der sozialen Verantwortung festigen heißt vor allem auch, sich immer wieder zu vergewissern, ob und wie die erwähnten Instrumente im Sinne der Goldenen Regel genutzt werden, gegebenenfalls auch geänderten Bedingungen angepasst werden müssen. Es bietet sich deshalb an, das in Kapitel 6.4.3, Controlling, beschriebene allgemeine Controlling um ein spezifisches „Verantwortungs-Controlling“ zu erweitern. Dieses unterstützt die Geschäftsführung und die übrigen Verantwortungsträger dabei, das Management der sozialen Verantwortung dauerhaft wirksam werden zu lassen.

Die Aufgaben des Verantwortungs-Controllings sind mit denen des Ethikkomitees in Teilen identisch. Diese Aufgaben werden hier im Sinne des Verantwortungs-Controllings ergänzt und detailliert. Dabei werden drei Teilfunktionen des Verantwortungs-Controllings unterschieden (in Anlehnung an Berkel; Herzog, S. 120): 
1. das Verantwortungs-Controlling als Planungsfunktion,

2. das operative Verantwortungs-Controlling und

3. das strategische Verantwortungs-Controlling

\section{Zu 1. das Verantwortungs-Controlling als Planungs- funktion}

Das Verantwortungs-Controlling ist eine Planungsfunktion. Es erarbeitet Empfehlungen zur Weiterentwicklung des Unternehmensleitbildes einschließlich der Führungsgrundsätze sowie der personellen und strukturellen Voraussetzungen für die Realisierung des besseren ManagementKonzepts.

Die Notwendigkeit zur Weiterentwicklung kann einerseits das Ergebnis der Kontrolltätigkeit sein (Näheres dazu in zu 2.). Die Initiative zur Weiterentwicklung des Management-Konzepts kann aber auch von Verantwortungsträgern ausgehen, die in eine Konflikt-Situation geraten sind, weil sich die Bedürfnisse der Stakeholder und/oder Aspekte der Handlungssituation des Krankenhausmanagements geändert haben. Die bisher aktuellen Werte und Normen und/oder die personellen und strukturellen Voraussetzungen sind zur Überwindung des entstandenen Konflikts nicht mehr geeignet.

\section{Zu 2. operatives Verantwortungs-Controlling}

Das operative Controlling stellt regelmäßig fest, ob die Verantwortungsträger sozial verantwortlich handeln oder ob sie Entscheidungen fällen, die mit der Goldenen Regel nicht im Einklang stehen - also Entscheidungen fällen, die von Stakeholdern nicht akzeptiert werden (können).

Wenn Differenzen zwischen dem von der Geschäftsführung angestrebten, nämlich konfliktfreien, und dem tatsächlichen Zustand festgestellt werden, gilt es die Gründe dafür zu finden. $\mathrm{Zu}$ diesen Gründen kann zum Beispiel das durch Fähigkeits- und/oder Wollen-Defizite bedingte Fehlverhalten von Verantwortungsträgern zählen. Es kommen aber auch die gegenüber den geltenden Vorgaben veränderten Bedeutungen der Stakeholder, veränderten Stakeholder-Bedürfnisse und deren Bewertungen und/oder die veränderten personellen und/oder institutionellen Voraussetzungen als Ursachen für fehlerhaftes Wahrnehmen von Verantwortung in Betracht. Wenn Verantwortungsträger nicht leitbildkonform gehandelt haben, wird auch das Unternehmensleitbild auf den Prüfstand gestellt werden müssen; es könnte ja sein, dass die in dem Unternehmensleitbild festgeschriebenen Werte und Normen nicht mehr zeitgemäß sind und deshalb abweichend von diesen gehandelt werden musste.

Einschlägige Korrekturen an

- den Inhalten des Unternehmensleitbildes,

- der Einstellung und den Fähigkeiten der betroffenen Verantwortungsträger,

- der Auswahl der zu berücksichtigenden Stakeholder und deren als legitim akzeptierten Bedürfnissen und/oder an

- den institutionellen Voraussetzungen für das Wahrnehmen von Verantwortung

müssen gegebenenfalls vorgenommen werden. Unter Berücksichtigung der Defizite und der Ursachen dafür werden Empfehlungen zur Weiterentwicklung des Management-Konzepts erarbeitet (s. Ziff. 1.) und der Geschäftsführung zur Beschlussfassung vorgelegt.

\section{Zu 3. strategisches Verantwortungs-Controlling}

Das strategische Verantwortungs-Controlling beobachtet die mittel- und langfristig zu erwartenden Entwicklungen der das Management der sozialen Verantwortung prägenden Faktoren. Das sind vor allem die Art und die Stärke des möglichen Einflusses der Stakeholder auf unternehmerische Entscheidungen, die Wichtigkeit der Stakeholder-Beiträge für das Krankenhaus sowie die Bedürfnisse der Stakeholder. Zu diesen Faktoren zählen aber auch Aspekte der Handlungssituation des Krankenhausmanagements, deren Veränderungen die Geschäftsführung möglicherweise zu einer Änderung der Vorgaben für die Berücksichtigung der Stakeholder und der Stakeholder-Bedürfnisse veranlasst (s. Beispiel).

\section{Beispiel}

Es ist seit vielen Jahren zu beobachten, dass sich der Staat seiner Verpflichtung, die Investitionen der Krankenhäuser durch Fördermittel zu finanzieren, immer mehr entzieht (Näheres dazu in Kap. 1.1). Angesichts der aktuellen wirtschaftlichen Entwicklung muss damit gerechnet werden, dass das Verhalten des Staates sich in Zukunft nicht ändern wird, dass die Höhe der den Krankenhäusern zur Verfügung gestellten Fördermittel eher noch deutlich geringer werden wird.

Die Geschäftsführung muss deshalb prüfen, mit welchem Gewicht die Bedürfnisse verschiedener Stakeholder - 
betroffen sind vor allem die Patienten, die Führungskräfte und Mitarbeiter, der Eigentümer sowie die Krankenkassen - bei unternehmerischen (Investitions-) Entscheidungen künftig berücksichtigt werden sollen. Soll auf die dringend notwendige Renovierung der Patientenzimmer zugunsten der Erneuerung medizin-technischer Geräte verzichtet werden? Müssen die Mitarbeiter weiter auf angemessene Gehaltszuwächse oder gar auf Teile des aktuellen Gehalts verzichten, um Gewinne als Grundlage für die Finanzierung von Investitionen erzielen zu können? Muss der Eigentümer seine Rendite-Erwartungen nach unten korrigieren, weil Teile des Gewinns in die Weiterentwicklung des Krankenhauses investiert werden müssen? Oder muss der Basisfallwert zu Lasten der Krankenkassen erhöht werden?

\subsubsection{Das Konzept festigen mittels kurzfristiger Erfolge}

An anderer Stelle wurde schon darauf hingewiesen, dass die Umstellung auf das neue Management-Konzept mehrere Jahre in Anspruch nehmen kann. In dieser Zeit werden Kritiker das Konzept immer wieder in Frage stellen. Sie werden fragen, ob die Geschäftsführung mit dem Prozess des Wandelns auf dem richtigen Kurs liegt. Wenn in dieser Phase überzeugende Erfolge als Belege für die Vernünftigkeit des neuen ManagementKonzepts nicht vorgelegt werden können, ist die Ablösung des bisherigen Management-Konzepts durch das der Goldenen Regel verpflichtete Konzept sehr bald zum Scheitern verurteilt (Kotter, S. 163).

Kurzfristige Erfolge sind überzeugende $\mathrm{Be}$ weise für die Richtigkeit der Veränderung. Sie erzeugen Glaubwürdigkeit und helfen der Geschäftsführung, die Belegschaft des Krankenhauses dauerhaft für den Transformationsprozess zu gewinnen (Kotter, S. 164). Kurzfristige Erfolge machen die Veränderungen erlebbar und lassen die Veränderungen als nützlich sowohl für die Verantwortungsträger als auch für die Stakeholder erkennbar werden; sie tragen auf diese Weise dazu bei, das Vertrauen der Verantwortungsträger in die Vorteilhaftigkeit des neuen Management-Konzepts zu festigen und Vorbehalte gegenüber den Veränderungen abzubauen.

Überzeugend sind Erfolge dann, wenn sie wie folgt charakterisiert werden können (Kotter, S. 167):
- Die Maßnahmen können von allen Verantwortungsträgern als Realität wahrgenommen werden.

- Die Maßnahmen werden realisiert, wie sie in dem Umstellungskonzept in inhaltlicher Hinsicht geplant worden sind.

- Die Maßnahmen werden realisiert, wie sie in zeitlicher Hinsicht vorgesehen sind. Dies gilt vor allem für die Maßnahmen, die mit einer hohen zeitlichen Priorität geplant worden sind, und die bei zeitgerechter Realisierung als Beleg für die Vernünftigkeit des Konzepts dienen können.

- Die Maßnahmen werden akzeptiert; sie werden von niemandem in Frage gestellt.

\section{Beispiel}

Das in Kapitel 8.2.3 beschriebene Konzept der Personalbedarfsermittlung wird in geeigneter Form (zum Beispiel mittels der Hauszeitschrift) allen Führungskräften und Mitarbeitern des Krankenhauses zur Kenntnis gebracht, und zwar in einer Sprache, die von allen im Krankenhaus arbeitenden Menschen verstanden wird. Diese Mitteilung schließt den Termin ein, ab wann das Konzept als Gegenstromkonzept unter Beteiligung der betroffenen Führungskräfte und Mitarbeiter angewendet wird.

Wenn dieses Konzept für die nächste Planungsperiode so wie beschrieben eingesetzt wird, ist das ein Erfolg, der die Umstellung auf das neue Management-Konzept sicher unterstützen wird.

Die Vorteile kurzfristiger Erfolge für die Festigung des besseren Management-Konzepts als Bestandteil des Krankenhaus-Alltags werden in Tabelle 28 zusammengefasst.

Tab. 28 Die Bedeutung kurzfristiger Erfolge nach Kotter (1997, S. 169)

Sie liefern den Beweis dafür, dass sich die großen Anstrengungen lohnen.

Erfolge rechtfertigen die durch den Wandel verursachten Kosten.

\section{Sie belohnen die Vermittler des Wandelns mit Anerkennung.}

Nach vielen Mühen und harter Arbeit bauen positive Rückmeldungen Moral und Motivation auf.

Sie unterstützen die Feinabstimmung von Vision, Unternehmensleitbild und Strategien. 
Kurzfristige Erfolge vermitteln der Geschäftsführung konkrete Daten über die Durchsetzbarkeit ihrer Vorstellungen.

\section{Sie schalten Zyniker und eigennützige Gegner aus.}

Eindeutige Verbesserungen machen es für Menschen schwierig, den erforderlichen Wandel zu blockieren.

Sie sichern die Unterstützung durch die Führungskräfte.

Sie liefern den höheren Hierarchieebenen den Beweis dafür, dass der Wandel planmäßig verläuft.

\section{Sie erzeugen Impulse.}

Sie verwandeln Neutrale in Befürworter, Widerwillige in Tatkräftige usw.

\subsection{Zusammenfassung und Checkliste}

Die Kapitel 6, 7 und 8.2 bis 8.7 beschreiben die Elemente des Managements der sozialen Verantwortung und den Weg des Wandelns zu diesem. Die Beispiele und Listen mit Anforderungen an die personellen und institutionellen Voraussetzungen können als Gebrauchsanweisung für die Umstellung verwendet werden.

Aus didaktischen Gründen werden die einzelnen Wegstrecken auf verschiedene Kapitel verteilt. Der Zusammenhang ist damit etwas verloren gegangen. Es bietet sich deshalb an, die notwendigen Schritte in einer Checkliste zusammenzufassen (s. Tab. 29).

Tab. 29 Der Prozess des Wandelns (Checkliste)

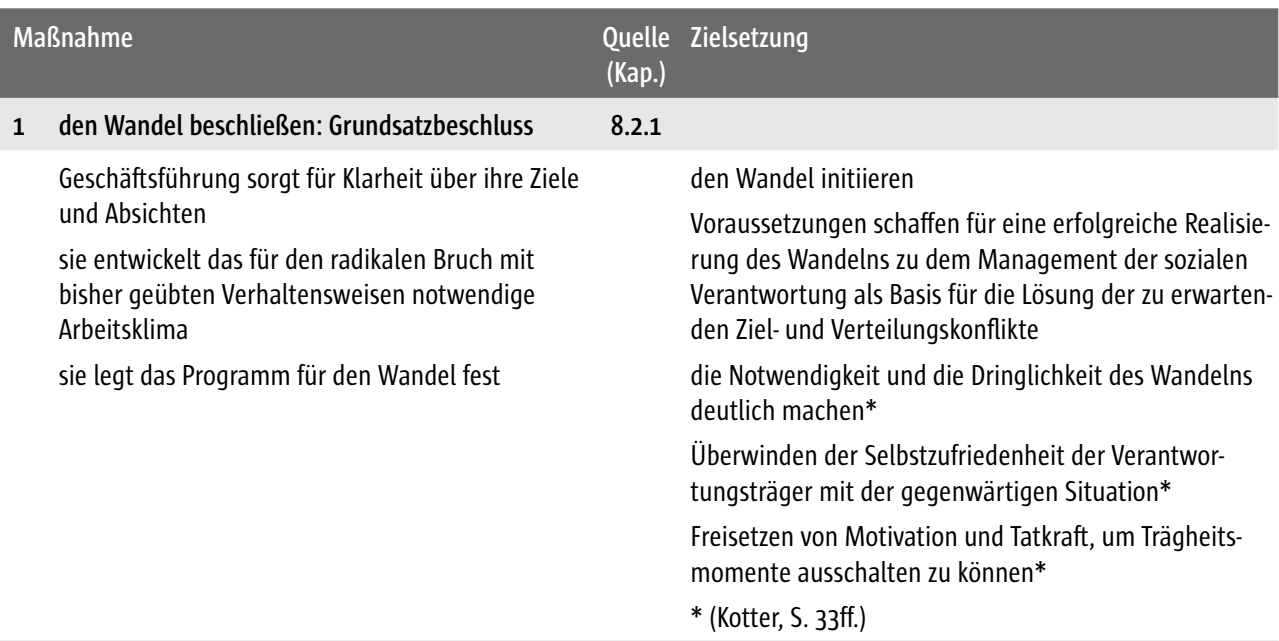

verantwortlich: Geschäftsführung

2 Organisation des Veränderungsprozesses

Beauftragung des Projektmanagement

Festlegen der Projekt-Organisation

und als Teile davon

- Sicherung der zur Umsetzung des Beschlusses erforderlichen Unterstützung durch Macht-Promotoren

- Bestellen eines fachkundigen Führungsteams zur Unterstützung der Geschäftsführung

- Aufteilung des Gesamtprojektes in Teil-Projekte
8.2.2

Unterstützung der Geschäftsführung bei der ProjektDurchführung

Sicherstellung der sach- und zeitgerechten Durchführung des Veränderungsprozesses 
Maßnahme

Quelle Zielsetzung

(Kap.)

3 Standortbestimmung

\subsection{Umweltanalyse auf Stakeholder-Basis}

Identifizierung und Charakterisierung der Stakeholder

Analyse, Bewertung und Gewichtung der Stakeholder-Anliegen einschließlich der Festlegung der dafür erforderlichen Bewertungskriterien
8.3

Ermittlung der Bedeutung der Stakeholder für das Krankenhaus, indem festgestellt wird,

- wie stark der Einfluss ist, den Stakeholder zur Durchsetzung ihrer Anliegen geltend machen können, und

- wie wichtig dem Krankenhaus die Beiträge sind, die die Stakeholder zur Sicherung der KrankenhausExistenz und zum erfolgreichen Betrieb des Krankenhauses leisten wollen,

und darauf aufbauend

Auswahl der bei Entscheidungen künftig zu berücksichtigenden Stakeholder

Ermittlung der Chancen und Risiken, die sich für das Unternehmen angesichts der Befriedigung oder Nicht-Befriedigung der zu beobachtenden StakeholderBedürfnisse ergeben,

und darauf aufbauend

Auswahl der Anliegen der ausgewählten Stakeholder, die künftig bei Entscheidungen berücksichtigt werden sollen.

verantwortlich: Verantwortungsträger, Projektmanager

\subsection{Unternehmensanalyse}

Analyse der Wahrnehmung von Verantwortung durch die Verantwortungsträger

Analyse der Voraussetzung für die Wahrnehmung von Verantwortung

- Eignung der organisatorischen Grundstruktur?

- Eignung der Verantwortungsträger?
8.4

Wie wichtig ist den Verantwortungsträgern die Berücksichtigung der Stakeholderanliegen?

Sind die personellen und strukturellen Voraussetzungen ausreichend, um sozial verantwortliches Handeln unterstützen zu können?

verantwortlich: Verantwortungsträger, Projektmanager

\section{4 den Wandel endgültig beschließen}

Aufbauend auf den Ergebnissen der Umwelt- und der Unternehmensanalyse und der Feststellung eventueller Defizite werden

- die Entwicklung bzw. Weiterentwicklung eines den Prinzipien der Goldenen Regel verpflichteten Unternehmensleitbildes beauftragt und realisiert,

- die Maßnahmen, die dem Schaffen der organisationalen Voraussetzungen für das Wahrnehmen von Verantwortung dienen sollen, identifiziert und deren Realisierung bzw. Weiterentwicklung veranlasst, und
8.2.3 mit dem Wandel unwiderruflich beginnen

Beseitigung eventuell vorhandener personeller und/ oder organisationaler Barrieren, die die Wahrnehmung von Verantwortung behindern könnten 
- die Maßnahmen identifiziert und deren Realisierung bzw. Weiterentwicklung veranlasst, die notwendig sind, um die Verantwortungsträger des Krankenhauses von der Nützlichkeit sozial verantwortlichen Handelns überzeugen und in den Stand versetzen zu können, dieses auch zu praktizieren.
7.3 und

verantwortlich: Geschäftsführung

5 Grundpostulate erarbeiten

6.2 Normen festlegen für die Entwicklung der

- der Unternehmensstruktur und

- des Unternehmensleitbildes

verantwortlich: Geschäftsführung, Verantwortungsträger

6 Das Management der sozialen Verantwortung realisieren

6.1 Verantwortung konkretisieren - Unternehmens- 8.7, leitbild erarbeiten - Unterstützung des Sollens $\quad 7.2$ und

Festlegen der Inhalte des Unternehmensleitbildes

5.3.2

Einführung des Unternehmensleitbildes

(Formulierung und Diffusion)

den Verantwortungsträgern deutlich machen, was unter sozial verantwortlichem Handeln verstanden wird, welche Handlungen erlaubt/erwünscht bzw. verboten/unerwünscht sind

die Voraussetzungen für die Zuschreibung von Verantwortung sind zu beachten

Festlegen der Werte und Normen, die für alle anderen Maßnahmen leitend sind

verantwortlich: Geschäftsführung, Verantwortungsträger, Projektmanager

6.2 für Verantwortungsbewusstsein sensibilisieren, Unterstützung des Wollens

die Belegschaft über den Fortschritt der Unternehmensleitbild-Formulierung informieren

Geschäftsführung präsentiert das Unternehmensleitbild

das Unternehmensleitbild wird zum Gegenstand der unternehmensinternen Fortbildung

die Konflikte, die die Krankenhausarbeit begleiten, identifizieren und diese als Argumentationsbasis analysieren

diverse Maßnahmen des Personalmanagements realisieren (Personalbeschaffung, -entwicklung als Einstellungsentwicklung und Personalführung)
8.6

7.3

die Verantwortungsträger davon überzeugen, dass es für das Krankenhaus (wie auch für sie selbst) und für die Stakeholder des Krankenhauses gleichermaßen von Nutzen ist, wenn sie die Normen des Unternehmensleitbildes bei allen ihren Entscheidungen und Handlungen anwenden.

die Verantwortungsträger dafür gewinnen, die ihnen zur Verfügung stehenden Freiheiten im Sinne der Goldenen Regel zu nutzen

verantwortlich: Geschäftsführung, Führungskräfte 
8 Was ist zu tun? Die Verankerung des Managements der sozialen Verantwortung im Krankenhaus

Maßnahme

Quelle Zielsetzung

(Kap.)

6.3 Unterstützung des Könnens

Unternehmensstruktur festlegen

Personalentwicklung im Sinne von Fähigkeits-

entwicklung

Installation eines Patientenfürsprechers und anderer Vertreter von Stakeholdern

Installation eines Ethikkomitees
6.4 Verantwortungsträger in die Lage versetzen, das

6.5 Konzept des Managements der sozialen Verantwortung

7.3 zu nutzen

7.4

7.4

verantwortlich: Geschäftsführung, Verantwortungsträger, Personalabteilung, Führungskräfte

7 Konzept des Managements der sozialen Verantwortung festigen

7.1 Verantwortungs-Controlling

8.8.2 Sicherstellen, dass das Konzept dauerhaft umgesetzt wird/werden kann

verantwortlich: Geschäftsführung, Controller

7.2 Kurzfristige Erfolge sicherstellen

8.8.3 Skeptiker von der Vorteilhaftigkeit des neuen

Managements-Konzepts überzeugen (Kotter, S. 164)

verantwortlich: Geschäftsführung, Projektmanager 